\title{
Real-Time Sweat pH Monitoring Based on a Wearable Chemical Barcode Micro-fluidic Platform Incorporating Ionic Liquids
}

\author{
Vincenzo F. Curto, ${ }^{\mathrm{a}, \mathrm{b}}$ Cormac Fay, ${ }^{\mathrm{a}}$ Shirley Coyle, ${ }^{\mathrm{a}}$ Robert Byrne, ${ }^{\mathrm{a}, \mathrm{b}}$ Corinne \\ O’Toole, ${ }^{\text {a,b }}$ Caroline Barry, ${ }^{\text {a,b }}$ Sarah Hughes, ${ }^{\text {a,c }}$ Niall Moyna, ${ }^{\text {a,c }}$ Dermot \\ Diamond, ${ }^{\mathrm{a}, \mathrm{b}}$ Fernando Benito-Lopez, ${ }^{* a, b}$
}

a CLARITY: Centre for Sensor Web Technologies, National Centre for Sensor Research, Glasnevin 9, Dublin, Ireland, Fax: +353 1700 7995; Tel: + 3531700 7603; E-mail: fernando.lopez@dcu.ie

${ }^{b}$ School of Chemical Sciences, Dublin City University, Dublin, Ireland.

${ }^{c}$ School of Health and Human Performance, Dublin City University, Dublin, Ireland.

\begin{abstract}
This work presents the fabrication, characterisation and the performance of a wearable, robust, flexible and disposable chemical barcode device based on a micro-fluidic platform that incorporates ionic liquid polymer gels (ionogels). The device has been applied to the monitoring of the $\mathrm{pH}$ of sweat in real time during an exercise period. The device is an ideal wearable sensor for measuring the $\mathrm{pH}$ of sweat since it does not contents any electronic part for fluidic handle or $\mathrm{pH}$ detection and because it can be directly incorporated into clothing, head- or wristbands, which are in continuous contact with the skin. In addition, due to the micro-fluidic structure, fresh sweat is continuously passing through the sensing area providing the capability to perform continuous real time analysis. The approach presented here ensures immediate feedback regarding sweat composition. Sweat analysis is attractive for monitoring purposes as it can provide physiological information directly relevant to the health and performance of the wearer without the need for an invasive sampling approach.
\end{abstract}

Corresponding author: Dr. Fernando Benito-Lopez. CLARITY: Centre for Sensor Web Technologies, National Centre for Sensor Research, Glasnevin 9, Dublin, Ireland, Fax: +353 1700 7995; Tel: + 353 1700 7603; E-mail: fernando.lopez@dcu.ie

Keywords: Sweat analysis, ionic liquid, ionogel, pH, wearable micro-fluidic. 


\section{Introduction}

Wearable sensors such as heart rate monitors and pedometers are in common use by people involved in sports and exercise activities. This area is growing exponentially, and while it is mainly driven by interest from health/sports enthusiasts, it will increasingly expand into health monitoring, as the economics of healthcare will force trends towards remote (home based) monitoring of patient status, rather than the current hospital focused model. In particular, the true potential of wearable chemical sensors for the real-time ambulatory monitoring of bodily fluids such as tears, sweat, urine and blood has not been realised due to difficulties associated with sample generation, collection and delivery, sensor calibration and reliability, wearability and safety issues.[1]

Sweat is naturally generated during exercise, thus the possibility of monitoring its contents provides very rich information about the physiological condition of the individual.[2] Sweat analysis is known to be used to identify pathological disorders such as cystic fibrosis.[3] Moreover, real-time sweat analysis, particularly during exercise,[4] potentially opens a route to gathering valuable information on dehydration and the detection of conditions related to changes in the concentrations of important biomolecules and ions, such as hyponatremia (low sodium concentration). This information can be used to optimise approaches to rehydration and remineralisation [5] which can enhance athletic performance and general health.

There are several factors that correlate the $\mathrm{pH}$ of sweat and health. Changes in the $\mathrm{pH}$ of the skin are reported to play a role in the pathogenesis of skin diseases like irritant contact dermatitis and acne, among others.[6] Patterson et al. showed that inducing metabolic alkalosis through the ingestion of sodium bicarbonate led to increased blood and sweat pH.[7] Furthermore, it has been reported that sweat $\mathrm{pH}$ will rise in response to an increased sweat rate.[8] A relationship was also observed between $\mathrm{pH}$ and sodium $\left(\mathrm{Na}^{+}\right)$levels in isolated sweat glands in that the greater the concentration of $\mathrm{Na}^{+}$, the higher the sweat $\mathrm{pH}$ will be.[9] As exercising in a dehydrated condition has been shown to lead to increased levels of $\mathrm{Na}^{+}$, it can be seen that such changes can be measured directly (using a $\mathrm{Na}^{+}$selective sensor) or indirectly by monitoring the $\mathrm{pH}$ of sweat.[10]

Micro-Total-Analysis ( $\mu$ TAS) or Lab-on-a-Chip (LOC) is an important concept for the development of personalised health care and point of care diagnostic devices, and 
it will improve the performance and capabilities of many commercial products that are already available in the market.[11] Important technological barriers such as miniaturisation, low cost production, reusability or disposability, robustness, flexibility and adaptability are continuously being overcome using this approach. However, sweat, which is easily accessible using non-invasive means, remains largely unexplored as a sample medium for tracking personal health status using the LOC approach.[12]

The use of optical $\mathrm{pH}$ sensors offer several advantages such as freedom from electrical noise, possibility of miniaturisation, ability to monitor status without physical contact, and flexibility in interrogation approaches (human eye, LEDsensors, cameras, spectrometers).[13] Also, optical pH sensors are suitable for applications where conventional electrodes cannot be used because of their size or because of the risk of electric shock, such as during in-vivo measurements. In order to provide optical $\mathrm{pH}$ sensors with good sensitivity, selectivity and stability, various support materials, methods and reagents, and immobilisation techniques for $\mathrm{pH}$ indicator dyes have been employed.[14,15] In particular, ionic liquids (ILs) have been rarely employed in optical sensors despite their excellent chemical and thermal stabilities, low vapour pressure, high ionic conductivity properties, and tuneable hydrophobic and hydrophilic nature.[16,17,18,19,20,21,22] The incorporation of ILs into polymer gels to form so-called 'ionogels' is a particularly attractive strategy as it may generate materials that maintain the inherent advantages of ILs within a solid or semi-solid gel-type structure.[23,24,25,26]

Here we show how a simple, autonomous, wearable, robust, flexible and disposable micro-fluidic platform based on ionogels can be used for monitoring the $\mathrm{pH}$ of sweat generated during an exercise period in real-time. Accurate $\mathrm{pH}$ values can be obtained by simply observing the barcode colour variation in comparison to a standard colour chart or through more sophisticated methods such as photo or video analysis of the colour changes. A significant advantage of these approaches is that the on-body sensor consumes no power, does not require any electronics for signal acquisition or communication, and therefore does not need a battery. On the other hand, remote interrogation by eye or by camera requires direct line of sight of the sensor status by the observer or camera. However, the colourimetric response can also be monitored on-line using simple opto-electronic components integrated into the device, along with wearable communications electronics (mote, dedicated platforms, mobile phones 
etc.), providing continuous feed-back of the sweat composition to remote locations via a local base-station.[12,26,27]

\section{Experimental}

\subsection{Materia/s}

$\mathrm{N}$-isopropylacrylamide (NIPAAm, Wako) was purified by recrystallisation in a mixed solution of hexane and toluene and dried under a vacuum. N,N-methylenebis(acrylamide) (MBAAm, Sigma Aldrich), 2,2-dimethoxy-2-phenyl acetophenone (DMPA, Sigma-Aldrich, St. Louis, USA), methyl red (MR), bromophenol blue (BPB), bromocresol green (BCG), bromocresol purple (BCP) and bromothymol blue (BTB) (Sigma-Aldrich, St. Louis, USA), were used without further purification. Trihexyltetradecyl-phosphonium dicyanoamide $\left[\mathrm{P}_{6,6,6,14}\right][\mathrm{dca}]$ were obtained with compliments of Cytec Industries. The IL was purified thoroughly by column chromatography, [28] dried under vacuum at $40^{\circ} \mathrm{C}$ for $48 \mathrm{~h}$, and stored under argon at $20^{\circ} \mathrm{C}$. Artificial sweat was prepared according to the standard ISO $3160-2(20 \mathrm{~g} / \mathrm{L}$ $\mathrm{NaCl}, 17.5 \mathrm{~g} / \mathrm{L} \mathrm{NH} 4 \mathrm{OH}, 5 \mathrm{~g} / \mathrm{L}$ acetic acid and $15 \mathrm{~g} / \mathrm{L}$ lactic acid) (Sigma-Aldrich, St. Louis, USA). Super-absorbent non-woven textiles (Absortex) were purchased from Texsus SpA, Italy. Hansaplast commercially available plasters were used to encapsulate the micro-fluidic platforms.

Devices were fabricated using multilayer lamination. $\mathrm{A} \mathrm{CO}_{2}$ laser (Laser Micromachining Light Deck, Optec, Belgium) system was used to cut the various polymer layers. Connecting holes and micro-fluidic channels were cut from an $80 \mu \mathrm{m}$ thick layer of pressure-sensitive adhesive (PSA - AR9808, Adhesives Research, Ireland) and laminated onto a $125 \mu \mathrm{m}$ poly(methylmethacrylate), PMMA, support layer (GoodFellow, UK) using a thermal roller laminator (Titan-110, GBC Films, USA).

Photographs were taken using a Canon PowerShot G7 camera. The skin $\mathrm{pH}$ sensor was purchased from Hanna Instruments, India. The UV light source used for photopolymerisation was a BONDwand UV-365 nm obtained from Electrolyte Corporation, USA. UV light intensity was measured with a Lutron (Taiwan) UV340A UV light meter. The pictures were processed and analysed using the Open Computer Vision (OpenCV) image processing libraries. 
The micro-fluidic platform $(20 \times 17 \mathrm{~mm})$, Fig. 1a, consists of four independent reservoirs and channels, fabricated in six layers of poly(methyl methacrylate) and PSA using $\mathrm{CO}_{2}$ ablation laser and lamination. Fig. $1 \mathrm{~b}$ shows the fabrication protocol that starts with a $125 \mu \mathrm{m}$ thick PMMA base layer followed by two layers of PSA (160 $\mu \mathrm{m})$, containing the micro-channels ( $5 \times 1 \mathrm{~mm}$ and $160 \mu \mathrm{m}$ in depth), four rectangular ionogel reservoirs and an absorbent reservoir. Then an additional layer of PMMA $(125 \mu \mathrm{m})$, which contains only the four rectangular reservoirs $(2 \times 6 \mathrm{~mm})$ and the absorbent reservoir, was laminated over the PSA layers. The incorporation of the four ionogels inside of the micro-fluidic was performed as describe in section 2.3. To seal the system, a lid consisting of two more layers, PSA and PMMA (205 $\mu \mathrm{m})$, was laminated over the previous four layers. The lid contains four rectangular holes $(1 \times 5$ $\mathrm{mm}$ ) fabricated using the $\mathrm{CO}_{2}$ laser. The holes were carefully arranged to site directly over the polymerised ionogels. The micro-channels connect the four rectangular independent ionogel/dyes reservoirs with a common reservoir $(15 \times 5 \mathrm{~mm}$ and 285 $\mu \mathrm{m}$ depth), where an absorbent fiber drives the sweat from the sensing area through the channels by capillary action. This ensured that fresh sweat from the skin is continuously drawn into contact with the ionogel/dyes sensors.

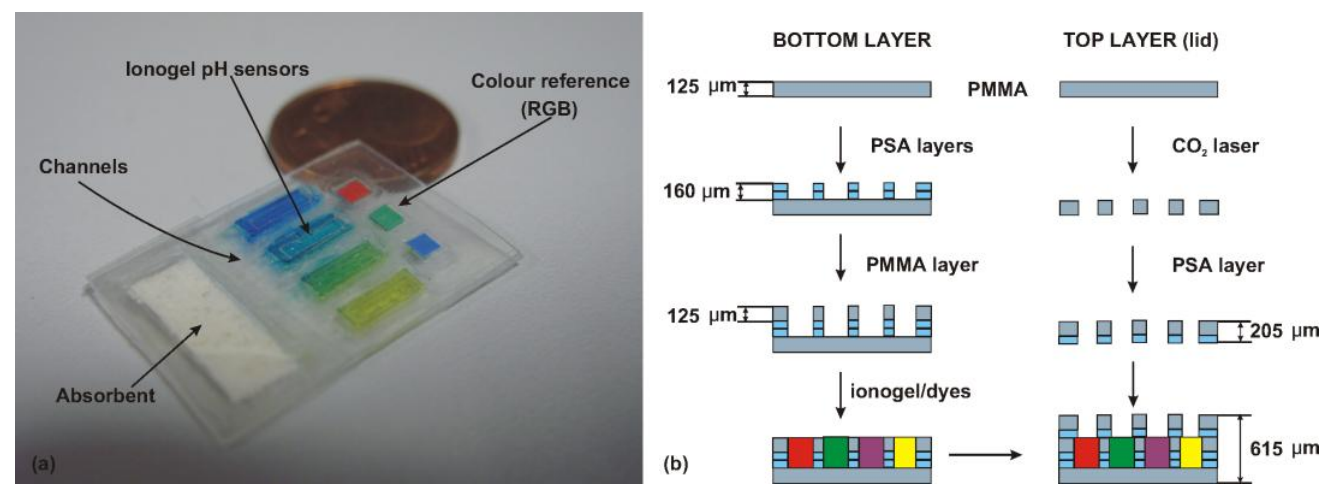

$<$ Fig. 1> 

Sensitive Dyes

The ionogel consisted of two monomeric units; $N$-isopropylacrylamide and $N, N^{\prime}-$ methylene-bis(acrylamide) in the ratio 100:5, respectively. The reaction mixture was prepared by dissolving the NIPAAm monomer $(4.0 \mathrm{mmol})$, the MBAAm $(0.2 \mathrm{mmol})$ and the photo-initiator 2,2-dimethoxy-2-phenylacetophenone DMPA $(0.11 \mathrm{mmol})$ into $1.5 \mathrm{~mL}$ of $\left[\mathrm{P}_{6,6,6,14}\right][\mathrm{dca}]$ ionic liquid, shown in Fig. $2 \mathrm{~b} .7 \mu \mathrm{L}$ of the reaction mixture was placed in each of the reservoirs after mixing at $45^{\circ} \mathrm{C}$ for 10 minutes. The monomers were then photo-polymerised within the ionic liquid matrix using a UV irradiation source $(365 \mathrm{~nm})$ placed $5 \mathrm{~cm}$ from the monomers for 30 minutes (UV intensity $\sim 630 \mu \mathrm{Wcm}^{-2}$ ). $365 \mathrm{~nm} \mathrm{UV}$ irradiation source is necessary to have the correct radical polymerisation process and obtain the desired ionogel structure and physical consistence. When the polymerisation was complete, the ionogels were washed with de-ionised water and subsequently with ethanol for 1 min each, and the procedure was repeated three times to remove any unpolymerised monomer and any excess of ionic liquid. Finally the ionogels were left to fully dry for five hours at room temperature. $5 \mu \mathrm{L}$ ethanol solution of each of the dyes $\left(10^{-3} \mathrm{M}\right)$ was pipetted over the ionogel and left until dry. This process was repeated three times. Then, the barcode system was washed in ethanol and in water several times until no leaching of the dyes was observed. The lid was placed on top of the barcode and laminated to form the final structure. Finally the device was dried at room temperature for five hours. The stability of the micro-fluidic barcode against $\mathrm{pH}$ was carried out following the same protocol than that in Benito-Lopez et al., and similar results were obtained. [26]
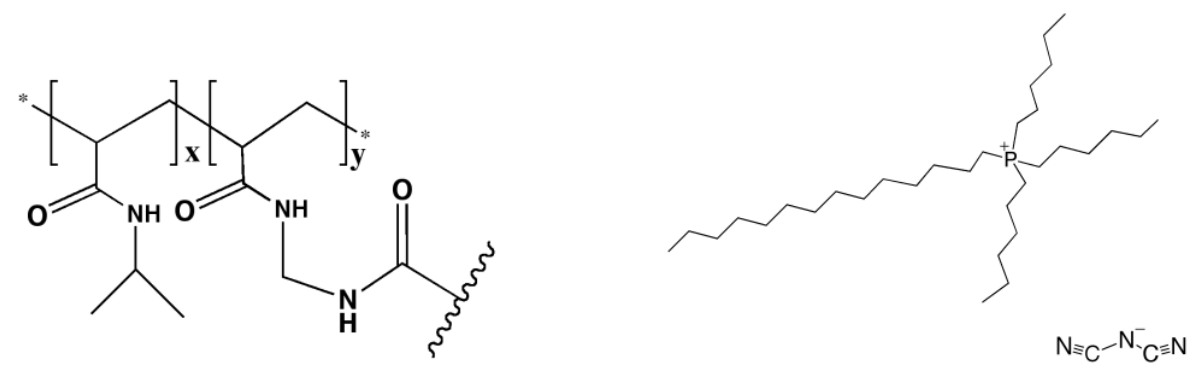

(a)

(b)

<Fig. 2> 


\section{4. pH Sensor and Optical Detection}

A colorimetric approach was employed to quantitatively determine the concentration of $\mathrm{pH}$ of sweat by means of a digital colour camera (Canon PowerShot G7). The approach involved the use of four $\mathrm{pH}$ sensitive dyes (methyl red, bromocresol green, bromocresol purple and bromothymol blue), which change in colour over a $\mathrm{pH}$ range defined by their respective pKa's. Fig. 3 visually shows colour changes in the dyes in a $\mathrm{pH}$ range from 4.5 to 8 , which covers the typical $\mathrm{pH}$ range of a human's sweat during exercise [8], i.e. from 5-7. Although this range was sufficiently covered, it was important to ascertain how the dyes respond over the full $\mathrm{pH}$ scale for later analysis. Therefore a calibration routine was carried out, where the platform was exposed to artificial sweat at different $\mathrm{pH}$, from ca. 1 to $c a$. 14 , within $0.5 \mathrm{pH}$ unit steps. A photograph of each event was captured with the parameters of the camera set to manual, 1/16 and at optimum resolution. For each capture, the camera was fixed at a distance of $5 \mathrm{~cm}$ from the barcode's planar surface along with ensuring that the barcode platform was captured in its entirety within the camera's field of view. In addition, a light source (60 W, Philips, 30*8L, Sportline R63, 240V, Holland) was placed at a $45^{\circ}$ angle for illumination and minimisation of background visual effects. Later, each captured image was processed by employing a standard set of algorithms using OpenCV. Firstly, each image region of interest (4 dyes and 3 reference patches) was identified through the creation of a binary mask image. This involved creating a copy of the original image, applying noise reduction filtering techniques (Gaussian blurring, median and erosion/dilation morphological algorithms) to aid in the segmentation step and then applying a connected component algorithm to the image. This resulted in a binary image with neighbouring pixels of similar colour being grouped together and identified as separate image regions. Next, the regions representing the 4 dyes and 3 reference patches were identified based on their location within the original image and stored in memory while the rest (misclassified regions) were omitted. After this, the resulting binary image was applied to the original image, removing the background (unwanted pixels) and leaving only pixel regions representing the ionogel/dye regions and reference patches. Subsequently, each region was considered in turn where the dominant colour component was calculated (i.e. the mean value) on each of the region's colour channel components (RGB). Next, the colour components of the dyes regions were normalised with respect to the 
reference patches to account for potential ambient lighting effects. A calibration plot for each dye was ascertained and the camera response ( $\left.\mathrm{R}^{\prime}\right)$ was calculated by: $\mathrm{R}^{\prime}=$ $\mathrm{R} /(\mathrm{R}+\mathrm{G}+\mathrm{B})$ using the normalised response of the RGB channels. Finally, a sigmoidal regression analysis (Boltzmann) was applied to achieve a calibration model.

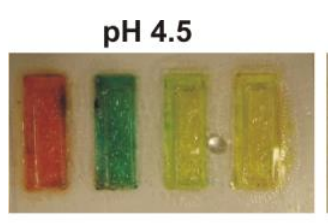

pH 6.5

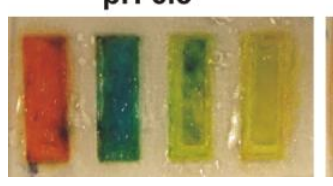

$\mathrm{pH} 5$

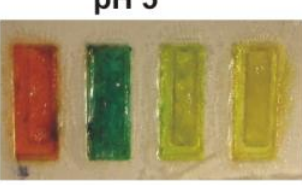

pH 7

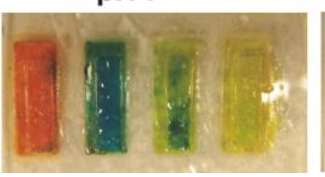

pH 5.5

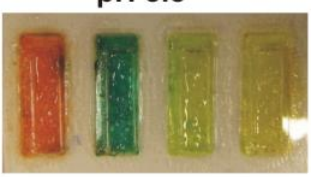

pH 7.5

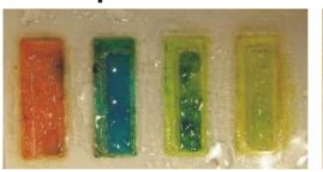

<Fig. 3>
pH 6

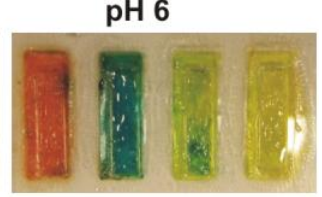

pH 8

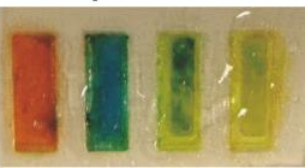

\subsection{On-Body Trials}

The micro-fluidic system was incorporated into an adhesive plaster to avoid direct contact of the ionogels with the skin, see Fig. 4a. The plaster was placed in the lower back region of the body where the sweat rate is approximately $0.85 \pm 0.41 \mathrm{mg} \mathrm{min}^{-1}$ $\mathrm{cm}^{-2}$.[9] Reference measurements were taken manually at fixed time intervals (10 min) using a commercial $\mathrm{pH}$ probe. At the same time three pictures of the barcode were taken in order to measure the $\mathrm{pH}$ of the sweat and for comparison with the reference values as explained above. The exercise protocol involved indoor cycling (room temperature $18{ }^{\circ} \mathrm{C}$ ) using a bicycle ergometer. Elite athletes participated in the study, who cycled for one hour at a self-selected pace.

\section{Results and Discussion}

\subsection{Why a Barcode pH sensor Micro-fluidic Platform?}

Several methods for measuring the $\mathrm{pH}$ of sweat are already established, which are based mainly on glass electrodes and ion-sensitive field-effect transistors (ISFET's). The most popular are planar-tipped conventional pH-probes, which can be placed directly in contact with the skin in order to measure the $\mathrm{pH}$. The drawback to this approach is that it is physically difficult to maintain contact between the probe and the skin over a prolonged period of time and it tends to suffer from drift and motion 
artefacts.

Moreover they are typically planar glass electrodes, which can cause skin damage when broken. The micro-fluidic platform is more fit-for-purpose as a wearable $\mathrm{pH}$ sensor since it can be directly incorporated into clothing or attached as an adhesive strip in continuous contact with the skin. Furthermore, due to the micro-fluidic structure, fresh sweat is continuously passing through the sensing area providing a real-time monitoring capability.

The ionogel matrix provides an ideal platform for the $\mathrm{pH}$ indicators dyes. This is because of, firstly, ion-pair interactions between the different $\mathrm{pH}$ indicators and the ionic liquid that forms the ionogel structure, and secondly, there is no leaching of the $\mathrm{pH}$ dyes during the experiments.[29] Furthermore, it was observed that the ionogel material is impressively robust under harsh conditions ( $\mathrm{pH}$ ranges from 0 to 14).[26]

\subsection{Micro-fluidic Platform Fabrication and Performance}

The micro-fluidic system was fabricated using six thin PMMA and PSA layers (615 $\mu \mathrm{m}$, total thickness). This ensures that the whole device is flexible and can easily adapt to the body contours. In addition, it is comfortable to wear providing an unobtrusive and non-invasive method for the analysis of sweat during exercise. The micro-fluidic system can be encapsulated into an adhesive plaster, Fig.4a, integrated in the sport clothes or into a sweat band worn on the head or the wrist, in order to directly obtain $\mathrm{pH}$ information of sweat during an exercise period, Fig. $4 \mathrm{~b}$.

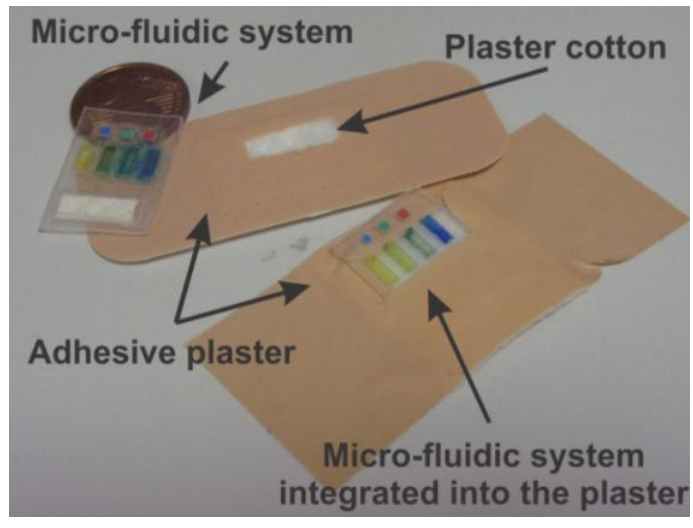

(a)

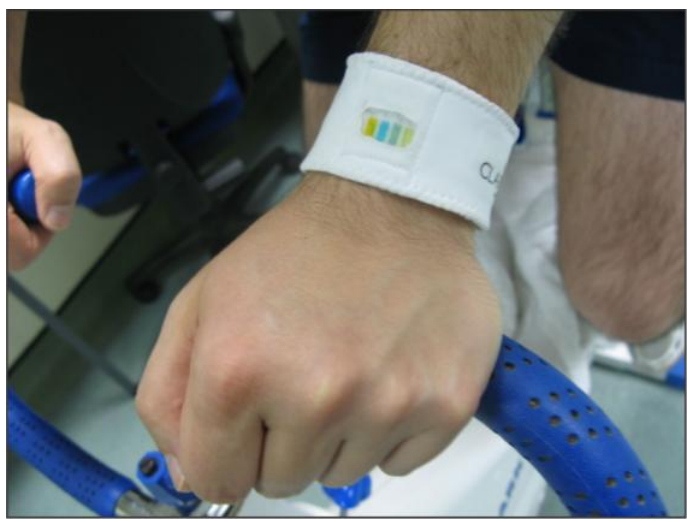

(b)

$<$ Fig. 4> 
The micro-fluidic structure ensures that fresh sweat is continuously sampled from the skin and flows pass the ionogels during the entire training period. The performance of the micro-fluidic platform is presented schematically in Fig.5a. Sweat is absorbed by the fabric of the clothes/adhesive plaster cotton and comes in contact with the barcode sensor. The dyes react with the sweat and change colour according to their respective pKa values. Sweat is continuously drawn through the micro-fluidic device by the super-absorbant material, which acts as a passive pump.

In order to test the performance of the micro-fluidic platform, artificial sweat was used to calculate the flow rate in the channels generated by the device. Snap-shot pictures of the channels were taken over time (see Fig.5b) and then analysed. The flow rate of the device was found to be initially ca. $6.4 \pm 2 \mu \mathrm{L} \mathrm{min}{ }^{-1}(\mathrm{n}=12)$ but once the micro-fluidic channel was filled up by the artificial sweat, the flow rate decreased gradually to $1.1 \pm 0.8 \mu \mathrm{L} \mathrm{min}{ }^{-1}(n=12)$ in the steady state. At this point, the flow rate remains constant until the absorbent reaches its maximum loading capacity, $148 \pm 2$ $\mu \mathrm{L}(\mathrm{n}=20)$. This gives the device an operational lifetime of ca. 135 minutes, in the current manifestation. However, since the device is easy to fabricate, and multiple replicates can be prepared in a single batch, the design can be easily modified for applications involving longer exercise periods. For example, the amount of absorbent material can be increased, or the channel dimensions varied to reduce the device flow rate, both of which would extend the useful operational time.

In addition, due to the inherent micro-sampling capability of the platform the area of the skin that is sampled is much smaller than commercially available sweat collection systems, i.e. patches. Considering the total exposed sensing areas, equal to the four lid holes $\left(4 \times 0.05 \mathrm{~cm}^{2}\right)$, the flow rate per unit area of the whole device is determined by the average steady state flow rate per unit area of each channel, $22 \mu \mathrm{L} \mathrm{min}{ }^{-1} \mathrm{~cm}^{-2}$, times four. This gives a total device flow rate of $88 \mu \mathrm{L} \mathrm{min}^{-1} \mathrm{~cm}^{-2}$. This value is much smaller than typical skin sweat flow rates, e.g. lower back $850 \pm 410 \mu \mathrm{L} \mathrm{cm}^{-2} \mathrm{~min}^{-1}$ (assuming sweat density equal to $1 \mathrm{mg} \mathrm{mL}^{-1}$ ).[9] This ensures that sufficient fresh sweat is always passing through the device. 


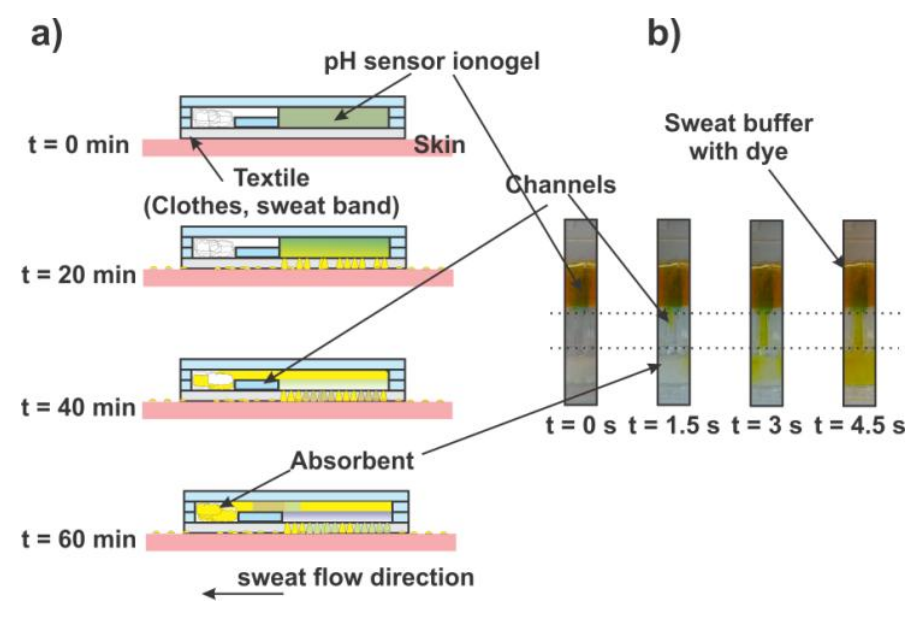

$<$ Fig. 5>

The response of the four immobilised dyes in the ionogel matrixes was evaluated through a calibration routine using buffer solutions, as explained in detail in section 2.4. The results show that the dyes exhibited a colour change depending on the $\mathrm{pH}$ and are shown in Fig.6. The change in colour intensity of each of the $\mathrm{pH}$ indicators was plotted against the $\mathrm{pH}$ value. A sigmoidal regression analysis (Boltzmann technique) was then applied to the calibration points and resulted in a calibration model for each dye.

Fig. 6 shows the calibration curves for the indicators BCG and BCP and as an example. The pKa of MR was not determined since its colour did not vary over the experimental $\mathrm{pH}$ range conditions. This could be due to the fact that the anion of the ionic liquid [dca]", that is known to show characteristics of Lewis base, [30] and this could interfere with the acid/base chemistry of the methyl red dye. For the other ionogel/dyes the experimental values for the pKa values were estimated to be: bromocresol green BCG: 3.43; bromocresol purple BCP: 7.61 and bromothymol blue BTB: 8.82 , which slightly varied with respect to the literature values (BCG: 4.6, BCP: 6.4 and BTB: 7.1). The variations are not surprising, as it has been shown that immobilisation of acidochromic dyes leads to variations in $\mathrm{pKa}$ due to a change of local micro-environment.[31]

Moreover, the stability of the barcode was demonstrated by performing three calibrations using three different barcode platforms. Calibration showed good repeatability with relative standard deviation (R.S.D.) typically within $4 \%(n=3)$. This indicated that the $\mathrm{pH}$ indicator dyes are fully reversible to $\mathrm{pH}$ changes and that no significant dye leaching occurred during the experiments. Signal intensity is 
reproducible after three calibrations using the same barcode with relative standard deviation (R.S.D.) typically within $6 \%(n=3)$.[26]
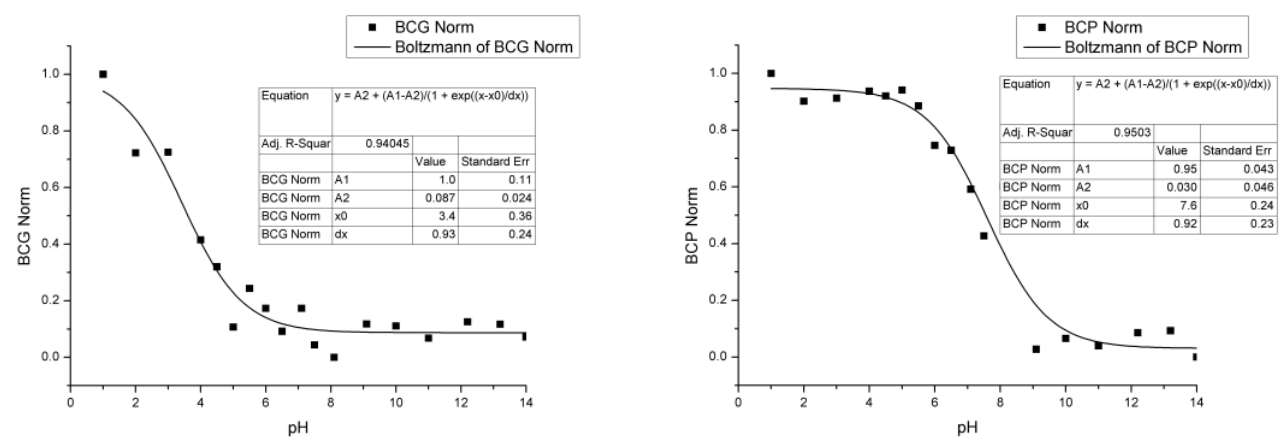

$<$ Fig. 6>

\subsection{On-body Trials}

Sweat flow rate and fluid losses vary for individuals and are generally dependent on body size, gender, exercise intensity, environmental conditions and individual metabolism.[32] For on-body trials, the subject was equipped with a micro-fluidic platform on the low back region. The micro-fluidic platform was activated before with a hydrochloric acid solution at $\mathrm{pH} 2$ for $5 \mathrm{~min}$. After a period of 20 minutes, following the approach used by Morris et al. where it was shown that it takes approximately 10-15 min to produce an appreciable amount of sweat during exercise [33], sweat reached the sensors and it was possible to begin monitoring the $\mathrm{pH}$ of the sweat. This delay arises firstly from the fact that sweat does not commence immediately upon exercise and that the device has a small but finite dead volume that must be filled before the sample reaches the sensors and a colour change is gained. Then a picture of the micro-fluidic platform was taken every $10 \mathrm{~min}$ along with parallel manual reference measurements using a $\mathrm{pH}$ electrode for specific use (Hanna instruments HI-1413B/50). The results are presented in Fig. 7-a. In the micro-fluidic platform, continuous fresh sweat is passed through the ionogel matrix, and the conditioning of the activation solution is quickly flushed away from the sensing area. After twenty minutes of a training period, no activation solution is observed in bromocresol green and bromocresol purple doped ionogels. For instance, the bromocresol green ionogel is yellow at times from 0 to 10 corresponding to a $\mathrm{pH} 2$ 
(i.e. that of the conditioning solution), after a 20 minute training period, the ionogel is blue in colour $(\mathrm{pH} 6)$ and it varies from dark to light blue, i.e. $\mathrm{pH}$ 5.5-6.5, during the rest of the experiment. Therefore, the $\mathrm{pH}$ of the two ionogels compared reasonably well with the commercial $\mathrm{pH}$ probe reference measurements.

As previously described, the ionogel incorporating the methyl red indicator did not perceptibly change over the whole $\mathrm{pH}$ range of study even though it has a $\mathrm{pK}_{\mathrm{a}}$ of 5 , (red to yellow). Therefore the dye was replaced by bromophenol blue that has a similar $\mathrm{pK}_{\mathrm{a}}(\sim 4)$ and it changes colour during the calibration process. Unfortunately, since the $\mathrm{pH}$ range of the dye is 3-4.6 (yellow to blue) a colour change gradient was not observed during trials. Moreover, no colour changes were also observed for the ionogel doped with bromothymol blue since the estimated $\mathrm{pK}_{\mathrm{a}}$ of 8.82 is over the range of the $\mathrm{pH}$ measurements carried out during the on-body trial shown in Fig. 7-a. Nevertheless, these two dyes (BPB, BTB) are potentially useful for picking up anomalous variations of the $\mathrm{pH}$ in the sweat during real-time analysis.

A more sophisticated approach to quantify the colour variations within the sweat's $\mathrm{pH}$ can be achieved using wearable device such as SMD-LED technology as previously reported. [26] However, a colourimetric electronic-free device can be easily read by the individual during the physical activity, considerably decreasing the complexity of the detection system (electronic part of the device) but improving the wearability and the read-out approach. Furthermore, the micro-fluidic platform has a major advantage in performance with respect to commercially available systems since they measure the $\mathrm{pH}$ of sweat from where it emerges and within an almost enclosed package therefore it minimises the interaction with carbon dioxide of the atmosphere, which can cause a lowering of the $\mathrm{pH}$ values.

In the presented system, a particular colour pattern of the barcode corresponds to a defined $\mathrm{pH}$ of the sweat where the captured images were analysed as explained earlier in Section 2.4 using OpenCV. Here, each $\mathrm{pH}$ prediction of each dye is calculated by normalisation with respect to the reference patches and then applied to the calibration model ascertained earlier. To achieve a single $\mathrm{pH}$ prediction from sensor barcode, each dye was considered equally with a weight of 1 and cumulative $\mathrm{pH}$ prediction was determined via their average value; this is shown in Fig. $7 \mathrm{~b}$ and values are presented in Table 1. It can be seen that by combining the two dyes a low 
relative percentage error was achieved with the exception of the first measurement (7.68\%) in where the dyes $\mathrm{pH}$ 's values might differ slightly from the ones of the $\mathrm{pH}$ meter due to residual conditioning of the activation solution in the ionogel. In addition, the Figure 7-b does show a similar trend by both measurement methods. It should be noted however that the accuracy of 0.49 of a $\mathrm{pH}$ unit ascertained in this study may need further investigation. For instance, a study may be needed to determine the correct weights when combining the dyes predictions to increase accuracy. However, to the best of our knowledge, the micro-fluidic device described in this work is the only wearable electronic-free sensor capable to perform real-time measurements during active exercise periods, with non-standardised light conditions. Similar work in the literature by Byrne et al. have reported an accuracy of $\pm 0.5 \mathrm{pH}$ units [34] when using a digital colour camera but under controlled lighting conditions.

Table 1. Time series measurements of $\mathrm{pH}$ from the reference instrument (pH meter) and the predictions of the dyes when combined and weighted equally. ${ }^{\dagger}$

\begin{tabular}{c|c|c|c} 
Time $[\mathrm{min}]$ & $\mathrm{pH}$ Meter & Dyes Prediction $(\mathrm{pH})$ & $\% \mathrm{RE}$ \\
\hline 20 & 6.38 & 5.89 & 7.68 \\
30 & 5.8 & 5.56 & 4.14 \\
40 & 5.67 & 5.67 & 0.00 \\
50 & 5.95 & 5.63 & 5.38
\end{tabular}

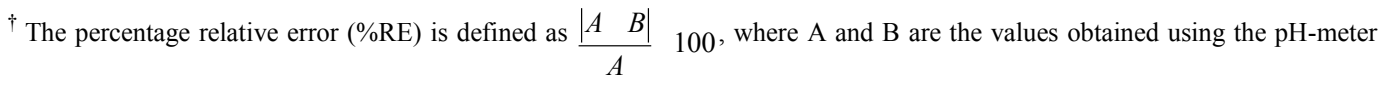
and the combined predictive values of the dyes, respectively.
} 

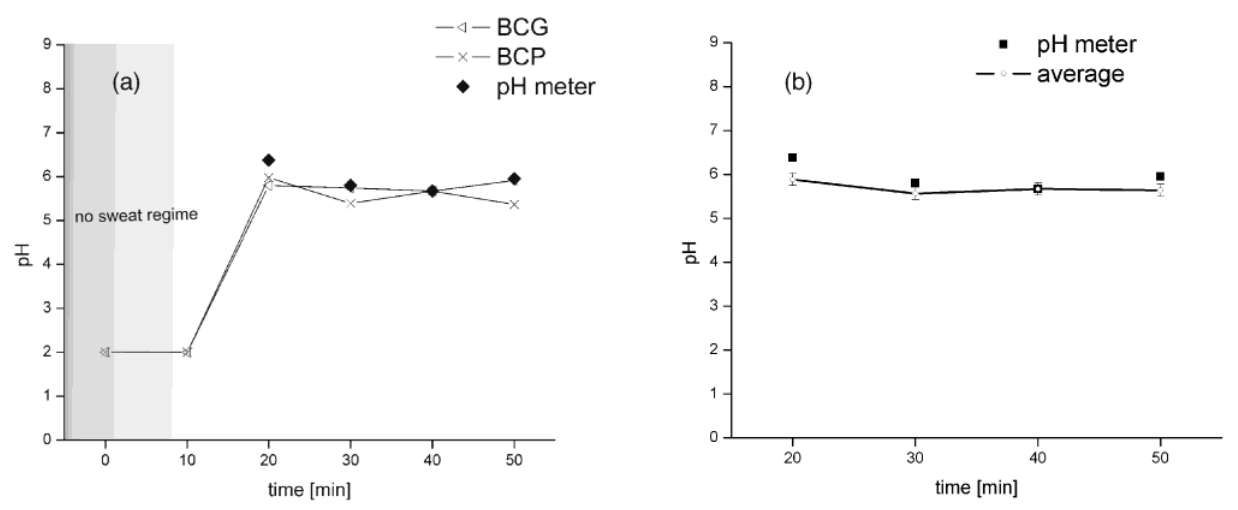

$<$ Fig. 7>

\section{Conclusions}

In this work, the fabrication, characterisation and the performance of a wearable, electronic-free and flexible micro-fluidic system based on ionic liquid polymer gels (ionogels) for monitoring in real-time the $\mathrm{pH}$ of the sweat generated during an exercise period has been presented.

As proven before, the ionogel matrix is very robust even at harsh $\mathrm{pH}$ conditions and that the $\mathrm{pH}$ indicators bromophenol blue, bromocresol green, bromocresol purple and bromothymol blue retained their $\mathrm{pH}$ indicator properties on the ionogel. The ionogeldye interactions ensure no leaching of the dyes during experiments, providing long durability of the device and accuracy on the $\mathrm{pH}$ of sweat measurements over time. The approach presented here provides immediate feedback regarding sweat composition, i.e. $\mathrm{pH}$, to individuals during exercise period. A particular colour pattern of the barcode corresponds to a defined $\mathrm{pH}$ of the sweat with an accuracy of $\sim 0.49 \mathrm{pH}$ units after applying standard image processing and analysis techniques to the pictures, which were captured during exercise trials when the sensor was applied on the skin. Future work will focus on the development of a more robust code for image processing, aiming a better resolution and accuracy in the $\mathrm{pH}$ prediction. Moreover, through a systematic comparison and correlation of $\mathrm{pH}$ of sweat with $\mathrm{pH}$ and lactate from blood, it will provide an easy, non-invasive and cheap tool to perform $\mathrm{pH}$ sweat analysis, improving sport performance and health.

\section{Acknowledgements}


This work was supported by Science Foundation Ireland under grant 07/CE/I1147 and the Research Career Start Programme 2010 fellowship form Dublin City University.

\section{Notes and references}

[1] Diamond D, Coyle S, Scarmagnani S, Hayes J (2008) Wireless sensor networks and chemo/biosensing. Chem Rev 108 (2):652-679

[2] Beauchamp M, Lands LC (2005) Sweat-testing: A review of current technical requirements. Pediatr Pulmonol 39 (6):507-511

[3] Jonsdottir B, Bergsteinsson H, Baldursson O (2008) Cystic Fibrosis - Review. Laeknabladid 94 (12):831-837

[4] Weber J, Kumar A, Kumar A, Bhansali S (2006) Novel lactate and pH biosensor for skin and sweat analysis based on single walled carbon nanotubes. Sens Actuators B 117 (1):308-313

[5] Casa DJ, Armstrong LE, Hillman SK, Montain SJ, Reiff RV, Rich BSE, Roberts WO, Stone JA (2000) National Athletic Trainers' Association position statement: Fluid replacement for athletes. J Athl Train 35 (2):212-224

[6] Schmid-Wendtner MH, Korting HC (2006) The pH of the skin surface and its impact on the barrier function. Skin Pharmacol Physiol 19 (6):296-302

[7] Patterson MJ, Galloway SDR, Nimmo MA (2002) Effect of induced metabolic alkalosis on sweat composition in men. Acta Physiol Scand 174 (1):41-46

[8] Granger D, Marsolais M, Burry J, Laprade R (2003) Na+/H+ exchangers in the human eccrine sweat duct. Am J Physiol-Cell Physiol 285 (5):C1047-C1058

[9] Patterson MJ, Galloway SDR, Nimmo MA (2000) Variations in regional sweat composition in normal human males. Exp Physiol 85 (6):869-875

[10] Morgan RM, Patterson MJ, Nimmo MA (2004) Acute effects of dehydration on sweat composition in men during prolonged exercise in the heat. Acta Physiol Scand 182 (1):37-43

[11] Whitesides G (2010) Solving problems. Lab Chip 10 (18):2317-2318

[12] Benito-Lopez F, Coyle S, Byrne R, Smeaton A, O'Connor NE, Diamond D (2009) Pump Less Wearable Microfluidic Device for Real Time $\mathrm{pH}$ Sweat Monitoring. Procedia Chem 1:1103-1106

[13] Diamond D (2004) Internet-scale sensing. Anal Chem 76 (15):278A-286A

[14] O'Toole M, Shepherd R, Wallace GG, Diamond D (2009) Inkjet printed LED based pH chemical sensor for gas sensing. Anal Chim Acta 652 (1-2):308-314

[15] Smyth C, Lau KT, Shepherd RL, Diamond D, Wu Y, Spinks GM, Wallace GG (2008) Selfmaintained colorimetric acid/base sensor using polypyrrole actuator. Sens Actuators B 129 (2):518-524 [16] Safavi A, Maleki N, Bagheri M (2007) Modification of chemical performance of dopants in xerogel films with entrapped ionic liquid. J Mater Chem 17 (17):1674-1681

[17] Seddon K (2002) Ionic liquids: designer solvents for green synthesis. TCE (730):33-35

[18] Byrne R, Coleman S, Fraser KJ, Raduta A, MacFarlane DR, Diamond D (2009) Photochromism of nitrobenzospiropyran in phosphonium based ionic liquids. Phys Chem Chem Phys 11 (33):72867291

[19] Byrne R, Fraser KJ, Izgorodina E, MacFarlane DR, Forsyth M, Diamond D (2008) Photo- and solvatochromic properties of nitrobenzospiropyran in ionic liquids containing the [NTf2]- anion. Phys Chem Chem Phys 10 (38):5919-5924

[20] Coleman S, Byrne R, Minkovska S, Diamond D (2009) Investigating Nanostructuring within Imidazolium Ionic Liquids: A Thermodynamic Study Using Photochromic Molecular Probes. The J Phys Chem B 113 (47):15589-15596

[21] Rogers RD, Seddon KR, Editors (2002) Ionic Liquids: Industrial Applications for Green Chemistry. (Proceedings of a Symposium held 1-5 April 2001 in San Diego, California.) [In: ACS Symp. Ser., 2002; 818.

[22] Wasserscheid P (2003) Recent developments in using ionic liquids as solvents and catalysts for organic synthesis. Organic Synthesis Highlights V:105-117

[23] Benito-Lopez F, Byrne R, Raduta AM, Vrana NE, McGuinness G, Diamond D (2010a) Ionogelbased light-actuated valves for controlling liquid flow in micro-fluidic manifolds. Lab Chip 10 (2):195201

[24] Neouze MA, Le Bideau J, Gaveau P, Bellayer S, Vioux A (2006) Ionogels, new materials arising from the confinement of ionic liquids within silica-derived networks. Chem Mater 18 (17):3931-3936 [25] Tamada M, Watanabe T, Horie K, Ohno H (2007) Control of ionic conductivity of ionic liquid/photoresponsive poly( amide acid) gels by photoirradiation. Chem Commun:4050-4052 
[26] Benito-Lopez F, Coyle S, Byrne R, O'Toole C, Barry C, Diamond D (2010b) Simple Barcode System Based on Inonogels for Real Time $\mathrm{pH}$-Sweat Monitoring. In: 7th International Workshop on Wearable and Implantable Body Sensor Networks (BSN 2010), 7-9 June 2010. pp 291-296

[27] Curto VF, Coyle S, Byrne R, Angelov N, Diamond D, Benito-Lopez F (2011) Concept and Development of an Autonomous Wearable Micro-fluidic Platform for real time $\mathrm{pH}$ Sweat Analysis, Sens Actuators B, Accepted.

[28] Ramnial T, Taylor S A, Bender M L, Gorodetsky B, Lee P T K, Dickie D A, McCollum B M, Pye C C, Walsby C J, Clyburne J A C (2008) Carbon-Centered Strong Bases in Phosphonium Ionic Liquids. J. Org. Chem. 73(3): 801-812

[29] O'Neill S, Conway S, Twellmeyer J, Egan O, Nolan K, Diamond D (1999) Ion-selective optode membranes using 9-(4-diethylamino-2-octadecanoatestyryl)-acridine acidochromic dye. Anal Chim Acta 398 (1):1-11

[30] MacFarlane DR, Pringle JM, Johansson KM, Forsyth SA, Forsyth M (2006) Lewis base ionic liquids. Chem. Commun. 1905-1917.

[31] Soller BR (1994) Design of intravascular fiber optic blood-gas sensors. IEEE Eng. Med. Biol. Mag. 13 (3): 327-335.

[32] A.I.S. Department of Sports Nutrition Data Sheet (2004) How much do athletes sweat? 1-5

[33] Morris D, Coyle S, Wu YZ, Lau KT, Wallace G, Diamond D (2009) Bio-sensing textile based patch with integrated optical detection system for sweat monitoring. Sens Actuators B 139 (1):231-236

[34] Byrne L, Lau K, Edwards S, Diamond D (2001) Digital imaging as a detector for quantitative colorimetric analyses, Proc. SPIE 4205, 267, doi:10.1117/12.417460. 


\section{Biographies}

Vincenzo Fabio Curto studied chemical engineering at University of Palermo, Italy (MSc Hons 2010). In 2010 he joined the Adaptive Sensors Group at Dublin City University where she is currently pursuing a PhD degree under the supervision of Prof. Dermot Diamond and Dr. Fernando BenitoLopez. His research interests include the development of wearable micro-fluidic system to perform real-time analysis.

Cormac Fay received a B.Eng. degree in Mechatronics and a M.Eng. degree in Telecommunications engineering, both from Dublin City University (DCU), Ireland, in 2005 and 2007, respectively. He subsequently pursued an internship within the Adaptive Information Cluster (AIC) in 2007 before progressing towards a research assistant position as part of the AIC and soon afterwards with the CLARITY Research Centre, DCU. His research area includes a range of disciplines including: novel environmental monitoring techniques, ultra low-power low cost environmental chemical sensing platforms, end-to-end system architectures, vision systems, wearable sensors, robotics, etc. His position continuously demands realizing the transition from chemical sensing to information retrieval via the world-wide-web.

Shirley Coyle is a researcher/designer in the field of wearable technologies and smart textiles. She has combined expertise in Biomedical Engineering and Fashion Design. She received her BEng in Electronic Engineering in 2000 from Dublin City University, Ireland. She then worked in the Information and Communications division in Siemens Ltd. for 2 years before commencing a Ph.D. study to develop the first optical brain computer interface. She received her PhD from the National University of Ireland Maynooth in 2005. Studying by night she graduated from the Grafton Academy of Fashion Design in 2008. She has worked on the EU FP6 'Biotex' project, a European-wide multipartner research effort to merge sensing capabilities with fabrics and textiles. She currently works within CLARITY: Centre for Sensor Web Technologies investigating ways to improve personal health and fitness using textile technologies.

Fernando Benito López studied chemistry at the Universidad Autonoma de Madrid and completed his master studies in the Department of Inorganic Chemistry in 2002. He obtained his PhD at the University of Twente, The Netherlands, under the supervision of Prof. David N. Reinhoudt and Dr. Willem Verboom in 2007. He carried out his postdoctoral research in the group of Prof. Dermot Diamond at Dublin City University, Dublin, Ireland. From 2010, he is Team Leader in polymer microfluidics at CLARITY: Centre for Sensor Web Technology, National Centre for Sensor Research, Dublin City University.

Robert Byrne studied pure and applied chemistry at Dublin City University (BSc Hons 2004) and received his PhD from Dublin City University (Materials Chemistry, 2008). His research interests include controlling molecular actuation by external stimuli for biomimetic applications.

Dermot Diamond received his PhD and DSc from Queen's University Belfast (Chemical Sensors, 1987, Internet Scale Sensing, 2002), and was VP for Research at Dublin City University (2002-2004). He has published over 200 peer-reviewed papers in international journals, is a named inventor in 13 patents, and is co-author and editor of three books. He is currently director of the National Centre for Sensor Research (www.ncsr.ie) and a Principle Investigator in CLARITY (www.clarity-centre.com/), a major research initiative focused on wireless sensor networks. In 2002 he was awarded the inaugural silver medal for Sensor Research by the RSC, London.

*Corresponding author: Tel.: +353 17007603 fax: +3531 7007995 .

E-mail addresses: fernando.lopez@dcu.ie (F. Benito-Lopez), vincenzo.curto2@mail.dcu.ie (V.F.Curto), dermot.diamond@dcu.ie (D. Diamond). 


\section{Figure Captions}

Fig. 1. (a) Picture of the micro-fluidic platform. (b) Micro-fluidic platform fabrication process.

Fig. 2. The molecular structure of the two components that make up the ionogel material. (a) $\mathrm{N}$ isopropylacrylamide and $N, N$-methylene-bis(acrylamide) crosslinked polymer in the ratio 100(x):5(y), and (b) the ionic liquid trihexyltetradecyl- phosphonium dicyanoamide $\left[\mathrm{P}_{6,6,6,14}\right][\mathrm{dca}]$ structure.

Fig. 3. Photographs of the micro-fluidic system at different pH's tested with artificial sweat (ISO 3160 2).

Fig. 4. Picture of the micro-fluidic system integrated into a plaster (a) and into a wrist-band (b).

Fig. 5. a) Schematic representation of the micro-fluidic system's performance over time. b) Series of pictures showing the channel performance in the micro-fluidic system (artificial sweat with dye), Pictures like these were used to estimate the sweat flow rate through the device.

Fig. 6. Calibration curves showing $\mathrm{pH} v \boldsymbol{s} \cdot \mathrm{R}^{\prime}=\mathrm{R} /(\mathrm{R}+\mathrm{G}+\mathrm{B})$ normalised $[0,1]$ a) bromocresol green and b) bromocresol purple.

Fig. 7. $\mathrm{pH}$ determination of sweat using the micro-fluidic system during a 50 min training period. (a) Plot showing the reference instrument in conjunction with the individual predictions of each dye when normalised with respect to the reference patches and predicted using the calibration model. (b) Plot of the reference measurement and the average of all the dye predictions when weighted equally. 


\title{
Real-Time Sweat pH Monitoring Based on a Wearable Chemical Barcode Micro-fluidic Platform Incorporating Ionic Liquids
}

\author{
Vincenzo F. Curto, ${ }^{\mathrm{a}, \mathrm{b}}$ Cormac Fay, ${ }^{\mathrm{a}}$ Shirley Coyle, ${ }^{\mathrm{a}}$ Robert Byrne, ${ }^{\mathrm{a}, \mathrm{b}}$ Corinne \\ O’Toole, ${ }^{\text {a,b }}$ Caroline Barry, ${ }^{\text {a,b }}$ Sarah Hughes, ${ }^{\text {a,c }}$ Niall Moyna, ${ }^{\text {a,c }}$ Dermot \\ Diamond, ${ }^{\mathrm{a}, \mathrm{b}}$ Fernando Benito-Lopez, ${ }^{* a, b}$
}

a CLARITY: Centre for Sensor Web Technologies, National Centre for Sensor Research, Glasnevin 9, Dublin, Ireland, Fax: +3531700 7995; Tel: + 3531700 7603; E-mail: fernando.lopez@dcu.ie

${ }^{b}$ School of Chemical Sciences, Dublin City University, Dublin, Ireland.

${ }^{c}$ School of Health and Human Performance, Dublin City University, Dublin, Ireland.

\begin{abstract}
This work presents the fabrication, characterisation and the performance of a wearable, robust, flexible and disposable chemical barcode device based on a micro-fluidic platform that incorporates ionic liquid polymer gels (ionogels). The device has been applied to the monitoring of the $\mathrm{pH}$ of sweat in real time during an exercise period. The device is an ideal wearable sensor for measuring the $\mathrm{pH}$ of sweat since it does not contents any electronic part for fluidic handle or $\mathrm{pH}$ detection and because it can be directly incorporated into clothing, head- or wristbands, which are in continuous contact with the skin. In addition, due to the micro-fluidic structure, fresh sweat is continuously passing through the sensing area providing the capability to perform continuous real time analysis. The approach presented here ensures immediate feedback regarding sweat composition. Sweat analysis is attractive for monitoring purposes as it can provide physiological information directly relevant to the health and performance of the wearer without the need for an invasive sampling approach.
\end{abstract}

Corresponding author: Dr. Fernando Benito-Lopez. CLARITY: Centre for Sensor Web Technologies, National Centre for Sensor Research, Glasnevin 9, Dublin, Ireland, Fax: +353 1700 7995; Tel: + 353 1700 7603; E-mail: fernando.lopez@dcu.ie

Keywords: Sweat analysis, ionic liquid, ionogel, pH, wearable micro-fluidic. 


\section{Introduction}

Wearable sensors such as heart rate monitors and pedometers are in common use by people involved in sports and exercise activities. This area is growing exponentially, and while it is mainly driven by interest from health/sports enthusiasts, it will increasingly expand into health monitoring, as the economics of healthcare will force trends towards remote (home based) monitoring of patient status, rather than the current hospital focused model. In particular, the true potential of wearable chemical sensors for the real-time ambulatory monitoring of bodily fluids such as tears, sweat, urine and blood has not been realised due to difficulties associated with sample generation, collection and delivery, sensor calibration and reliability, wearability and safety issues.[1]

Sweat is naturally generated during exercise, thus the possibility of monitoring its contents provides very rich information about the physiological condition of the individual.[2] Sweat analysis is known to be used to identify pathological disorders such as cystic fibrosis.[3] Moreover, real-time sweat analysis, particularly during exercise,[4] potentially opens a route to gathering valuable information on dehydration and the detection of conditions related to changes in the concentrations of important biomolecules and ions, such as hyponatremia (low sodium concentration). This information can be used to optimise approaches to rehydration and remineralisation [5] which can enhance athletic performance and general health.

There are several factors that correlate the $\mathrm{pH}$ of sweat and health. Changes in the $\mathrm{pH}$ of the skin are reported to play a role in the pathogenesis of skin diseases like irritant contact dermatitis and acne, among others.[6] Patterson et al. showed that inducing metabolic alkalosis through the ingestion of sodium bicarbonate led to increased blood and sweat pH.[7] Furthermore, it has been reported that sweat $\mathrm{pH}$ will rise in response to an increased sweat rate.[8] A relationship was also observed between $\mathrm{pH}$ and sodium $\left(\mathrm{Na}^{+}\right)$levels in isolated sweat glands in that the greater the concentration of $\mathrm{Na}^{+}$, the higher the sweat $\mathrm{pH}$ will be.[9] As exercising in a dehydrated condition has been shown to lead to increased levels of $\mathrm{Na}^{+}$, it can be seen that such changes can be measured directly (using a $\mathrm{Na}^{+}$selective sensor) or indirectly by monitoring the $\mathrm{pH}$ of sweat.[10]

Micro-Total-Analysis ( $\mu$ TAS) or Lab-on-a-Chip (LOC) is an important concept for the development of personalised health care and point of care diagnostic devices, and 
it will improve the performance and capabilities of many commercial products that are already available in the market.[11] Important technological barriers such as miniaturisation, low cost production, reusability or disposability, robustness, flexibility and adaptability are continuously being overcome using this approach. However, sweat, which is easily accessible using non-invasive means, remains largely unexplored as a sample medium for tracking personal health status using the LOC approach.[12]

The use of optical $\mathrm{pH}$ sensors offer several advantages such as freedom from electrical noise, possibility of miniaturisation, ability to monitor status without physical contact, and flexibility in interrogation approaches (human eye, LEDsensors, cameras, spectrometers).[13] Also, optical pH sensors are suitable for applications where conventional electrodes cannot be used because of their size or because of the risk of electric shock, such as during in-vivo measurements. In order to provide optical $\mathrm{pH}$ sensors with good sensitivity, selectivity and stability, various support materials, methods and reagents, and immobilisation techniques for $\mathrm{pH}$ indicator dyes have been employed.[14,15] In particular, ionic liquids (ILs) have been rarely employed in optical sensors despite their excellent chemical and thermal stabilities, low vapour pressure, high ionic conductivity properties, and tuneable hydrophobic and hydrophilic nature.[16,17,18,19,20,21,22] The incorporation of ILs into polymer gels to form so-called 'ionogels' is a particularly attractive strategy as it may generate materials that maintain the inherent advantages of ILs within a solid or semi-solid gel-type structure.[23,24,25,26]

Here we show how a simple, autonomous, wearable, robust, flexible and disposable micro-fluidic platform based on ionogels can be used for monitoring the $\mathrm{pH}$ of sweat generated during an exercise period in real-time. Accurate $\mathrm{pH}$ values can be obtained by simply observing the barcode colour variation in comparison to a standard colour chart or through more sophisticated methods such as photo or video analysis of the colour changes. A significant advantage of these approaches is that the on-body sensor consumes no power, does not require any electronics for signal acquisition or communication, and therefore does not need a battery. On the other hand, remote interrogation by eye or by camera requires direct line of sight of the sensor status by the observer or camera. However, the colourimetric response can also be monitored on-line using simple opto-electronic components integrated into the device, along with wearable communications electronics (mote, dedicated platforms, mobile phones 
etc.), providing continuous feed-back of the sweat composition to remote locations via a local base-station.[12,26,27]

\section{Experimental}

\subsection{Materia/s}

$\mathrm{N}$-isopropylacrylamide (NIPAAm, Wako) was purified by recrystallisation in a mixed solution of hexane and toluene and dried under a vacuum. N,N-methylenebis(acrylamide) (MBAAm, Sigma Aldrich), 2,2-dimethoxy-2-phenyl acetophenone (DMPA, Sigma-Aldrich, St. Louis, USA), methyl red (MR), bromophenol blue (BPB), bromocresol green (BCG), bromocresol purple (BCP) and bromothymol blue (BTB) (Sigma-Aldrich, St. Louis, USA), were used without further purification. Trihexyltetradecyl-phosphonium dicyanoamide $\left[\mathrm{P}_{6,6,6,14}\right][\mathrm{dca}]$ were obtained with compliments of Cytec Industries. The IL was purified thoroughly by column chromatography, [28] dried under vacuum at $40^{\circ} \mathrm{C}$ for $48 \mathrm{~h}$, and stored under argon at $20^{\circ} \mathrm{C}$. Artificial sweat was prepared according to the standard ISO $3160-2(20 \mathrm{~g} / \mathrm{L}$ $\mathrm{NaCl}, 17.5 \mathrm{~g} / \mathrm{L} \mathrm{NH} 4 \mathrm{OH}, 5 \mathrm{~g} / \mathrm{L}$ acetic acid and $15 \mathrm{~g} / \mathrm{L}$ lactic acid) (Sigma-Aldrich, St. Louis, USA). Super-absorbent non-woven textiles (Absortex) were purchased from Texsus SpA, Italy. Hansaplast commercially available plasters were used to encapsulate the micro-fluidic platforms.

Devices were fabricated using multilayer lamination. $\mathrm{A} \mathrm{CO}_{2}$ laser (Laser Micromachining Light Deck, Optec, Belgium) system was used to cut the various polymer layers. Connecting holes and micro-fluidic channels were cut from an $80 \mu \mathrm{m}$ thick layer of pressure-sensitive adhesive (PSA - AR9808, Adhesives Research, Ireland) and laminated onto a $125 \mu \mathrm{m}$ poly(methylmethacrylate), PMMA, support layer (GoodFellow, UK) using a thermal roller laminator (Titan-110, GBC Films, USA).

Photographs were taken using a Canon PowerShot G7 camera. The skin $\mathrm{pH}$ sensor was purchased from Hanna Instruments, India. The UV light source used for photopolymerisation was a BONDwand UV-365 nm obtained from Electrolyte Corporation, USA. UV light intensity was measured with a Lutron (Taiwan) UV340A UV light meter. The pictures were processed and analysed using the Open Computer Vision (OpenCV) image processing libraries. 


\subsection{Micro-fluidic Platform Fabrication}

The micro-fluidic platform $(20 \times 17 \mathrm{~mm})$, Fig. 1a, consists of four independent reservoirs and channels, fabricated in six layers of poly(methyl methacrylate) and PSA using $\mathrm{CO}_{2}$ ablation laser and lamination. Fig. $1 \mathrm{~b}$ shows the fabrication protocol that starts with a $125 \mu \mathrm{m}$ thick PMMA base layer followed by two layers of PSA (160 $\mu \mathrm{m})$, containing the micro-channels ( $5 \times 1 \mathrm{~mm}$ and $160 \mu \mathrm{m}$ in depth), four rectangular ionogel reservoirs and an absorbent reservoir. Then an additional layer of PMMA $(125 \mu \mathrm{m})$, which contains only the four rectangular reservoirs $(2 \times 6 \mathrm{~mm})$ and the absorbent reservoir, was laminated over the PSA layers. The incorporation of the four ionogels inside of the micro-fluidic was performed as describe in section 2.3. To seal the system, a lid consisting of two more layers, PSA and PMMA (205 $\mu \mathrm{m})$, was laminated over the previous four layers. The lid contains four rectangular holes $(1 \times 5$ $\mathrm{mm}$ ) fabricated using the $\mathrm{CO}_{2}$ laser. The holes were carefully arranged to site directly over the polymerised ionogels. The micro-channels connect the four rectangular independent ionogel/dyes reservoirs with a common reservoir $(15 \times 5 \mathrm{~mm}$ and 285 $\mu \mathrm{m}$ depth), where an absorbent fiber drives the sweat from the sensing area through the channels by capillary action. This ensured that fresh sweat from the skin is continuously drawn into contact with the ionogel/dyes sensors.

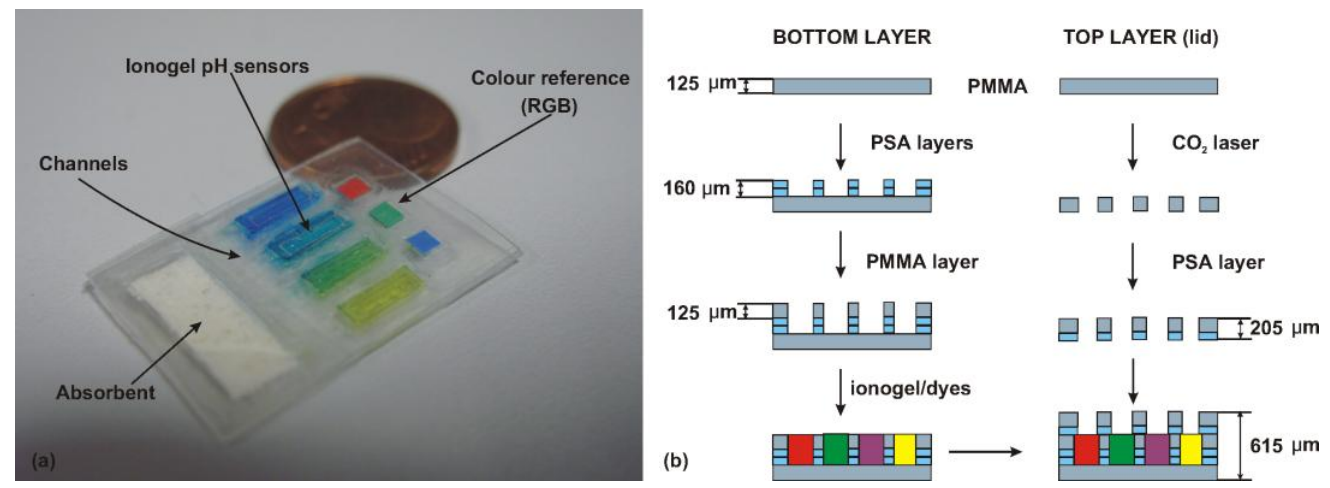

$<$ Fig. 1> 

Sensitive Dyes

The ionogel consisted of two monomeric units; $N$-isopropylacrylamide and $N, N^{\prime}-$ methylene-bis(acrylamide) in the ratio 100:5, respectively. The reaction mixture was prepared by dissolving the NIPAAm monomer $(4.0 \mathrm{mmol})$, the MBAAm $(0.2 \mathrm{mmol})$ and the photo-initiator 2,2-dimethoxy-2-phenylacetophenone DMPA $(0.11 \mathrm{mmol})$ into $1.5 \mathrm{~mL}$ of $\left[\mathrm{P}_{6,6,6,14}\right][\mathrm{dca}]$ ionic liquid, shown in Fig. $2 \mathrm{~b} .7 \mu \mathrm{L}$ of the reaction mixture was placed in each of the reservoirs after mixing at $45^{\circ} \mathrm{C}$ for 10 minutes. The monomers were then photo-polymerised within the ionic liquid matrix using a UV irradiation source $(365 \mathrm{~nm})$ placed $5 \mathrm{~cm}$ from the monomers for 30 minutes (UV intensity $\sim 630 \mu \mathrm{Wcm}^{-2}$ ). $365 \mathrm{~nm} \mathrm{UV}$ irradiation source is necessary to have the correct radical polymerisation process and obtain the desired ionogel structure and physical consistence. When the polymerisation was complete, the ionogels were washed with de-ionised water and subsequently with ethanol for 1 min each, and the procedure was repeated three times to remove any unpolymerised monomer and any excess of ionic liquid. Finally the ionogels were left to fully dry for five hours at room temperature. $5 \mu \mathrm{L}$ ethanol solution of each of the dyes $\left(10^{-3} \mathrm{M}\right)$ was pipetted over the ionogel and left until dry. This process was repeated three times. Then, the barcode system was washed in ethanol and in water several times until no leaching of the dyes was observed. The lid was placed on top of the barcode and laminated to form the final structure. Finally the device was dried at room temperature for five hours. The stability of the micro-fluidic barcode against $\mathrm{pH}$ was carried out following the same protocol than that in Benito-Lopez et al., and similar results were obtained. [26]
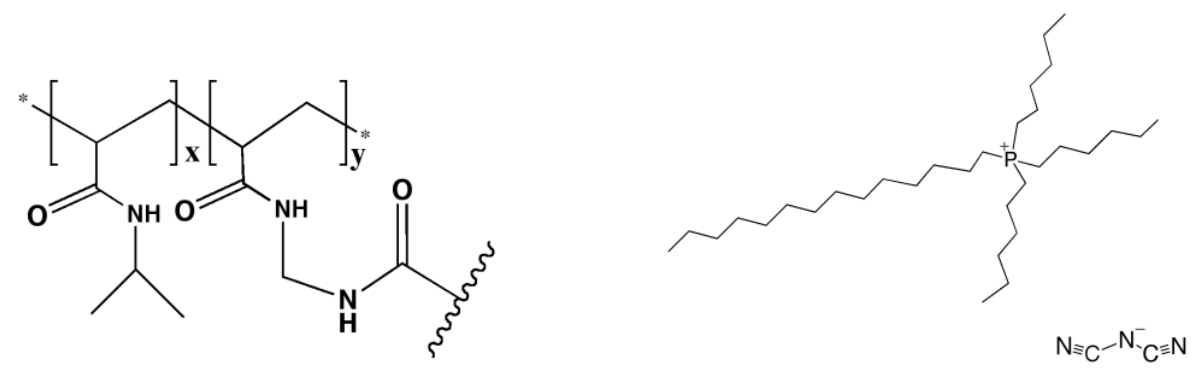

(a)

(b)

<Fig. 2> 


\section{4. pH Sensor and Optical Detection}

A colorimetric approach was employed to quantitatively determine the concentration of $\mathrm{pH}$ of sweat by means of a digital colour camera (Canon PowerShot G7). The approach involved the use of four $\mathrm{pH}$ sensitive dyes (methyl red, bromocresol green, bromocresol purple and bromothymol blue), which change in colour over a $\mathrm{pH}$ range defined by their respective pKa's. Fig. 3 visually shows colour changes in the dyes in a $\mathrm{pH}$ range from 4.5 to 8 , which covers the typical $\mathrm{pH}$ range of a human's sweat during exercise [8], i.e. from 5-7. Although this range was sufficiently covered, it was important to ascertain how the dyes respond over the full $\mathrm{pH}$ scale for later analysis. Therefore a calibration routine was carried out, where the platform was exposed to artificial sweat at different $\mathrm{pH}$, from ca. 1 to $c a .14$, within $0.5 \mathrm{pH}$ unit steps. A photograph of each event was captured with the parameters of the camera set to manual, 1/16 and at optimum resolution. For each capture, the camera was fixed at a distance of $5 \mathrm{~cm}$ from the barcode's planar surface along with ensuring that the barcode platform was captured in its entirety within the camera's field of view. In addition, a light source (60 W, Philips, 30*8L, Sportline R63, 240V, Holland) was placed at a $45^{\circ}$ angle for illumination and minimisation of background visual effects. Later, each captured image was processed by employing a standard set of algorithms using OpenCV. Firstly, each image region of interest (4 dyes and 3 reference patches) was identified through the creation of a binary mask image. This involved creating a copy of the original image, applying noise reduction filtering techniques (Gaussian blurring, median and erosion/dilation morphological algorithms) to aid in the segmentation step and then applying a connected component algorithm to the image. This resulted in a binary image with neighbouring pixels of similar colour being grouped together and identified as separate image regions. Next, the regions representing the 4 dyes and 3 reference patches were identified based on their location within the original image and stored in memory while the rest (misclassified regions) were omitted. After this, the resulting binary image was applied to the original image, removing the background (unwanted pixels) and leaving only pixel regions representing the ionogel/dye regions and reference patches. Subsequently, each region was considered in turn where the dominant colour component was calculated (i.e. the mean value) on each of the region's colour channel components (RGB). Next, the colour components of the dyes regions were normalised with respect to the reference 
patches to account for potential ambient lighting effects. A calibration plot for each dye was ascertained and the camera response $\left(R^{\prime}\right)$ was calculated by: $R^{\prime}=R /(R+G+B)$ using the normalised response of the RGB channels. Finally, a sigmoidal regression analysis (Boltzmann) was applied to achieve a calibration model.

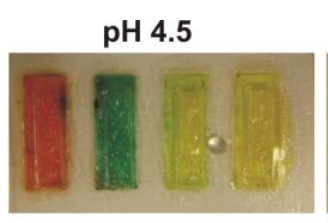

pH 6.5

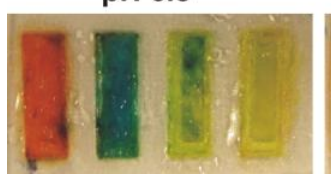

$\mathrm{pH} 5$

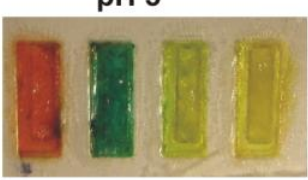

pH 7

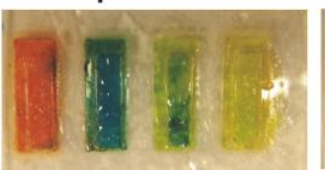

pH 5.5

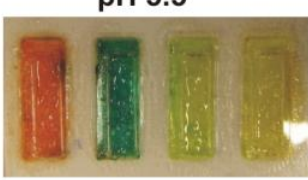

$\mathrm{pH} 7.5$

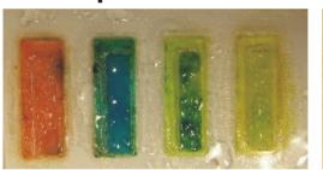

<Fig. 3>
$\mathrm{pH} 6$

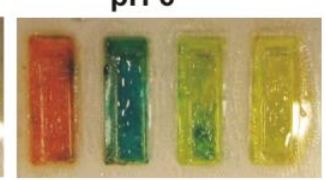

pH 8

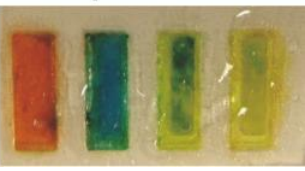

\subsection{On-Body Trials}

The micro-fluidic system was incorporated into an adhesive plaster to avoid direct contact of the ionogels with the skin, see Fig. 4a. The plaster was placed in the lower back region of the body where the sweat rate is approximately $0.85 \pm 0.41 \mathrm{mg} \mathrm{min}^{-1}$ $\mathrm{cm}^{-2}$.[9] Reference measurements were taken manually at fixed time intervals (10 min) using a commercial $\mathrm{pH}$ probe. At the same time three pictures of the barcode were taken in order to measure the $\mathrm{pH}$ of the sweat and for comparison with the reference values as explained above. The exercise protocol involved indoor cycling (room temperature $18{ }^{\circ} \mathrm{C}$ ) using a bicycle ergometer. Elite athletes participated in the study, who cycled for one hour at a self-selected pace.

\section{Results and Discussion}

\subsection{Why a Barcode pH sensor Micro-fluidic Platform?}

Several methods for measuring the $\mathrm{pH}$ of sweat are already established, which are based mainly on glass electrodes and ion-sensitive field-effect transistors (ISFET's). The most popular are planar-tipped conventional pH-probes, which can be placed directly in contact with the skin in order to measure the $\mathrm{pH}$. The drawback to this approach is that it is physically difficult to maintain contact between the probe and the skin over a prolonged period of time and it tends to suffer from drift and motion 
artefacts.

Moreover they are typically planar glass electrodes, which can cause skin damage when broken. The micro-fluidic platform is more fit-for-purpose as a wearable $\mathrm{pH}$ sensor since it can be directly incorporated into clothing or attached as an adhesive strip in continuous contact with the skin. Furthermore, due to the micro-fluidic structure, fresh sweat is continuously passing through the sensing area providing a real-time monitoring capability.

The ionogel matrix provides an ideal platform for the $\mathrm{pH}$ indicators dyes. This is because of, firstly, ion-pair interactions between the different $\mathrm{pH}$ indicators and the ionic liquid that forms the ionogel structure, and secondly, there is no leaching of the $\mathrm{pH}$ dyes during the experiments.[29] Furthermore, it was observed that the ionogel material is impressively robust under harsh conditions ( $\mathrm{pH}$ ranges from 0 to 14).[26]

\subsection{Micro-fluidic Platform Fabrication and Performance}

The micro-fluidic system was fabricated using six thin PMMA and PSA layers (615 $\mu \mathrm{m}$, total thickness). This ensures that the whole device is flexible and can easily adapt to the body contours. In addition, it is comfortable to wear providing an unobtrusive and non-invasive method for the analysis of sweat during exercise. The micro-fluidic system can be encapsulated into an adhesive plaster, Fig.4a, integrated in the sport clothes or into a sweat band worn on the head or the wrist, in order to directly obtain $\mathrm{pH}$ information of sweat during an exercise period, Fig. $4 \mathrm{~b}$.

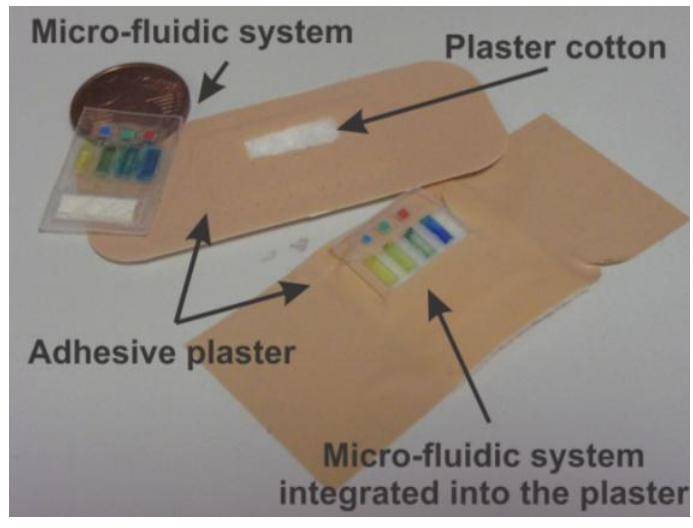

(a)

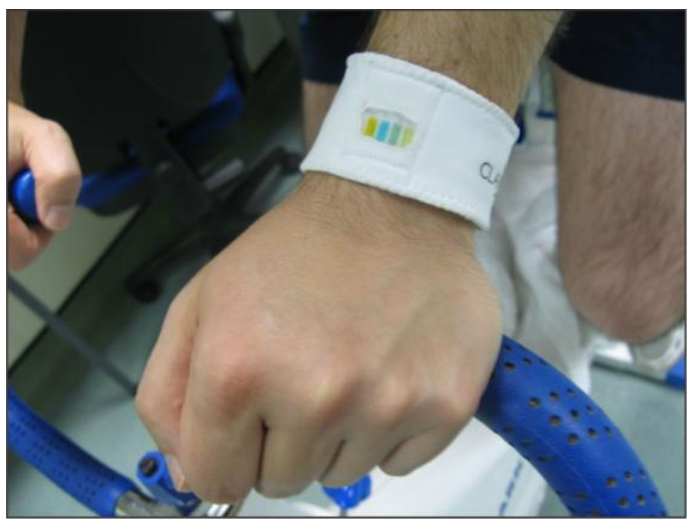

(b)

$<$ Fig. 4> 
The micro-fluidic structure ensures that fresh sweat is continuously sampled from the skin and flows pass the ionogels during the entire training period. The performance of the micro-fluidic platform is presented schematically in Fig.5a. Sweat is absorbed by the fabric of the clothes/adhesive plaster cotton and comes in contact with the barcode sensor. The dyes react with the sweat and change colour according to their respective pKa values. Sweat is continuously drawn through the micro-fluidic device by the super-absorbant material, which acts as a passive pump.

In order to test the performance of the micro-fluidic platform, artificial sweat was used to calculate the flow rate in the channels generated by the device. Snap-shot pictures of the channels were taken over time (see Fig.5b) and then analysed. The flow rate of the device was found to be initially ca. $6.4 \pm 2 \mu \mathrm{L} \mathrm{m^{-1 }}(\mathrm{n}=12)$ but once the micro-fluidic channel was filled up by the artificial sweat, the flow rate decreased gradually to $1.1 \pm 0.8 \mu \mathrm{L} \mathrm{min}{ }^{-1}(n=12)$ in the steady state. At this point, the flow rate remains constant until the absorbent reaches its maximum loading capacity, $148 \pm 2$ $\mu \mathrm{L}(\mathrm{n}=20)$. This gives the device an operational lifetime of ca. 135 minutes, in the current manifestation. However, since the device is easy to fabricate, and multiple replicates can be prepared in a single batch, the design can be easily modified for applications involving longer exercise periods. For example, the amount of absorbent material can be increased, or the channel dimensions varied to reduce the device flow rate, both of which would extend the useful operational time.

In addition, due to the inherent micro-sampling capability of the platform the area of the skin that is sampled is much smaller than commercially available sweat collection systems, i.e. patches. Considering the total exposed sensing areas, equal to the four lid holes $\left(4 \times 0.05 \mathrm{~cm}^{2}\right)$, the flow rate per unit area of the whole device is determined by the average steady state flow rate per unit area of each channel, $22 \mu \mathrm{L} \mathrm{min}{ }^{-1} \mathrm{~cm}^{-2}$, times four. This gives a total device flow rate of $88 \mu \mathrm{L} \mathrm{min}^{-1} \mathrm{~cm}^{-2}$. This value is much smaller than typical skin sweat flow rates, e.g. lower back $850 \pm 410 \mu \mathrm{L} \mathrm{cm}^{-2} \mathrm{~min}^{-1}$ (assuming sweat density equal to $1 \mathrm{mg} \mathrm{mL}^{-1}$ ).[9] This ensures that sufficient fresh sweat is always passing through the device. 


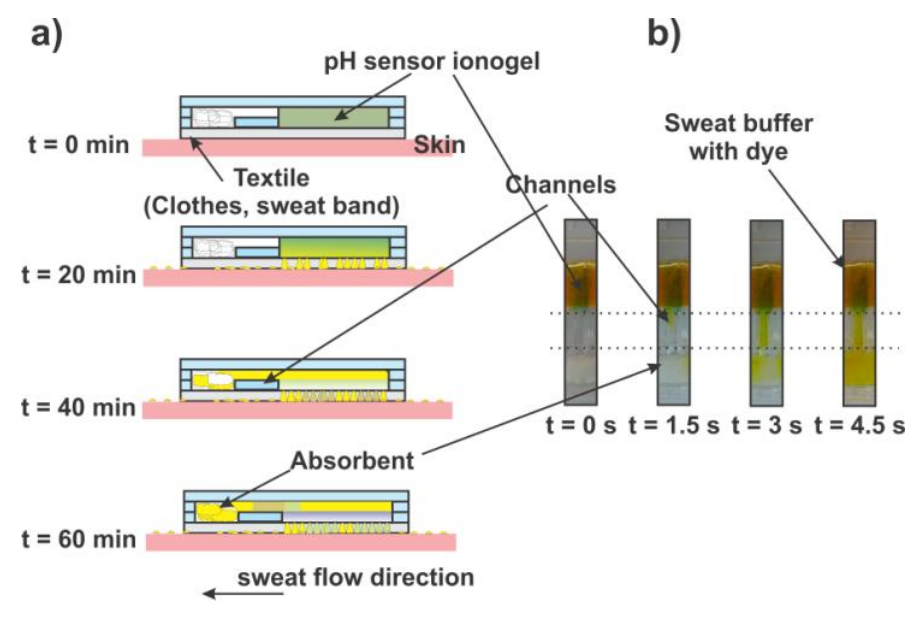

$<$ Fig. 5>

The response of the four immobilised dyes in the ionogel matrixes was evaluated through a calibration routine using buffer solutions, as explained in detail in section 2.4. The results show that the dyes exhibited a colour change depending on the $\mathrm{pH}$ and are shown in Fig.6. The change in colour intensity of each of the $\mathrm{pH}$ indicators was plotted against the $\mathrm{pH}$ value. A sigmoidal regression analysis (Boltzmann technique) was then applied to the calibration points and resulted in a calibration model for each dye.

Fig. 6 shows the calibration curves for the indicators BCG and BCP and as an example. The pKa of MR was not determined since its colour did not vary over the experimental $\mathrm{pH}$ range conditions. This could be due to the fact that the anion of the ionic liquid [dca], that is known to show characteristics of Lewis base, [30] and this could interfere with the acid/base chemistry of the methyl red dye. For the other ionogel/dyes the experimental values for the pKa values were estimated to be: bromocresol green BCG: 3.43; bromocresol purple BCP: 7.61 and bromothymol blue BTB: 8.82 , which slightly varied with respect to the literature values (BCG: 4.6, BCP: 6.4 and BTB: 7.1). The variations are not surprising, as it has been shown that immobilisation of acidochromic dyes leads to variations in $\mathrm{pKa}$ due to a change of local micro-environment.[31]

Moreover, the stability of the barcode was demonstrated by performing three calibrations using three different barcode platforms. Calibration showed good repeatability with relative standard deviation (R.S.D.) typically within $4 \%(n=3)$. This indicated that the $\mathrm{pH}$ indicator dyes are fully reversible to $\mathrm{pH}$ changes and that no significant dye leaching occurred during the experiments. Signal intensity is 
reproducible after three calibrations using the same barcode with relative standard deviation (R.S.D.) typically within $6 \%(n=3)$.[26]
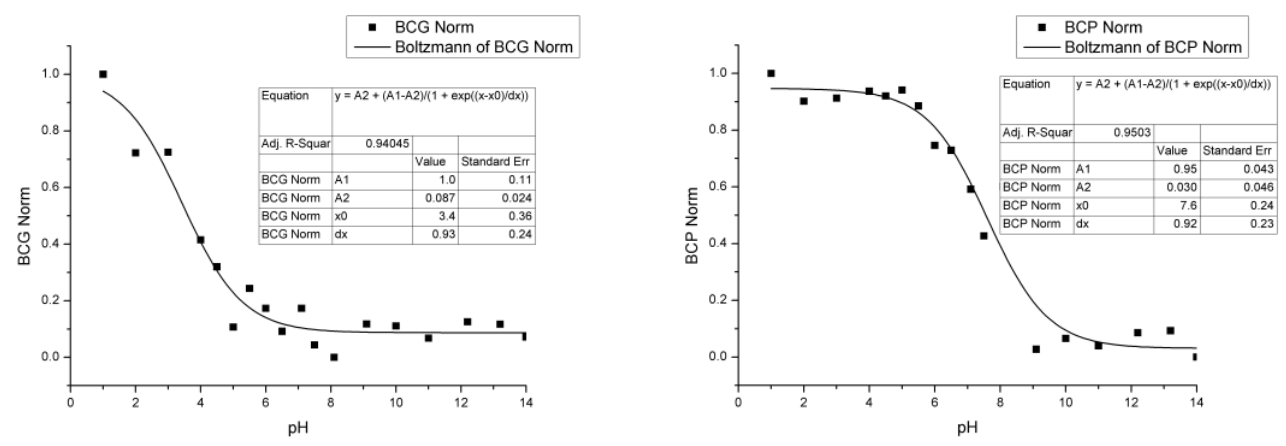

$<$ Fig. 6>

\subsection{On-body Trials}

Sweat flow rate and fluid losses vary for individuals and are generally dependent on body size, gender, exercise intensity, environmental conditions and individual metabolism.[32] For on-body trials, the subject was equipped with a micro-fluidic platform on the low back region. The micro-fluidic platform was activated before with a hydrochloric acid solution at $\mathrm{pH} 2$ for $5 \mathrm{~min}$. After a period of 20 minutes, following the approach used by Morris et al. where it was shown that it takes approximately 10-15 min to produce an appreciable amount of sweat during exercise [33], sweat reached the sensors and it was possible to begin monitoring the $\mathrm{pH}$ of the sweat. This delay arises firstly from the fact that sweat does not commence immediately upon exercise and that the device has a small but finite dead volume that must be filled before the sample reaches the sensors and a colour change is gained. Then a picture of the micro-fluidic platform was taken every $10 \mathrm{~min}$ along with parallel manual reference measurements using a $\mathrm{pH}$ electrode for specific use (Hanna instruments HI-1413B/50). The results are presented in Fig. 7-a. In the micro-fluidic platform, continuous fresh sweat is passed through the ionogel matrix, and the conditioning of the activation solution is quickly flushed away from the sensing area. After twenty minutes of a training period, no activation solution is observed in bromocresol green and bromocresol purple doped ionogels. For instance, the bromocresol green ionogel is yellow at times from 0 to 10 corresponding to a $\mathrm{pH} 2$ 
(i.e. that of the conditioning solution), after a 20 minute training period, the ionogel is blue in colour $(\mathrm{pH} 6)$ and it varies from dark to light blue, i.e. $\mathrm{pH}$ 5.5-6.5, during the rest of the experiment. Therefore, the $\mathrm{pH}$ of the two ionogels compared reasonably well with the commercial $\mathrm{pH}$ probe reference measurements.

As previously described, the ionogel incorporating the methyl red indicator did not perceptibly change over the whole $\mathrm{pH}$ range of study even though it has a $\mathrm{pK}_{\mathrm{a}}$ of 5 , (red to yellow). Therefore the dye was replaced by bromophenol blue that has a similar $\mathrm{pK}_{\mathrm{a}}(\sim 4)$ and it changes colour during the calibration process. Unfortunately, since the $\mathrm{pH}$ range of the dye is 3-4.6 (yellow to blue) a colour change gradient was not observed during trials. Moreover, no colour changes were also observed for the ionogel doped with bromothymol blue since the estimated $\mathrm{pK}_{\mathrm{a}}$ of 8.82 is over the range of the $\mathrm{pH}$ measurements carried out during the on-body trial shown in Fig. 7-a. Nevertheless, these two dyes (BPB, BTB) are potentially useful for picking up anomalous variations of the $\mathrm{pH}$ in the sweat during real-time analysis.

A more sophisticated approach to quantify the colour variations within the sweat's $\mathrm{pH}$ can be achieved using wearable device such as SMD-LED technology as previously reported. [26] However, a colourimetric electronic-free device can be easily read by the individual during the physical activity, considerably decreasing the complexity of the detection system (electronic part of the device) but improving the wearability and the read-out approach. Furthermore, the micro-fluidic platform has a major advantage in performance with respect to commercially available systems since they measure the $\mathrm{pH}$ of sweat from where it emerges and within an almost enclosed package therefore it minimises the interaction with carbon dioxide of the atmosphere, which can cause a lowering of the $\mathrm{pH}$ values.

In the presented system, a particular colour pattern of the barcode corresponds to a defined $\mathrm{pH}$ of the sweat where the captured images were analysed as explained earlier in Section 2.4 using OpenCV. Here, each $\mathrm{pH}$ prediction of each dye is calculated by normalisation with respect to the reference patches and then applied to the calibration model ascertained earlier. To achieve a single $\mathrm{pH}$ prediction from sensor barcode, each dye was considered equally with a weight of 1 and cumulative $\mathrm{pH}$ prediction was determined via their average value; this is shown in Fig. $7 \mathrm{~b}$ and values are presented in Table 1. It can be seen that by combining the two dyes a low 
relative percentage error was achieved with the exception of the first measurement (7.68\%) in where the dyes $\mathrm{pH}$ 's values might differ slightly from the ones of the $\mathrm{pH}$ meter due to residual conditioning of the activation solution in the ionogel. In addition, the Figure 7-b does show a similar trend by both measurement methods. It should be noted however that the accuracy of 0.49 of a $\mathrm{pH}$ unit ascertained in this study may need further investigation. For instance, a study may be needed to determine the correct weights when combining the dyes predictions to increase accuracy. However, to the best of our knowledge, the micro-fluidic device described in this work is the only wearable electronic-free sensor capable to perform real-time measurements during active exercise periods, with non-standardised light conditions. Similar work in the literature by Byrne et al. have reported an accuracy of $\pm 0.5 \mathrm{pH}$ units [34] when using a digital colour camera but under controlled lighting conditions.

Table 1. Time series measurements of $\mathrm{pH}$ from the reference instrument (pH meter) and the predictions of the dyes when combined and weighted equally. ${ }^{\dagger}$

\begin{tabular}{c|c|c|c} 
Time $[\mathrm{min}]$ & $\mathrm{pH}$ Meter & Dyes Prediction $(\mathrm{pH})$ & $\% \mathrm{RE}$ \\
\hline 20 & 6.38 & 5.89 & 7.68 \\
30 & 5.8 & 5.56 & 4.14 \\
40 & 5.67 & 5.67 & 0.00 \\
50 & 5.95 & 5.63 & 5.38
\end{tabular}

\footnotetext{
${ }^{\dagger}$ The percentage relative error (\%RE) is defined as $\frac{|A \quad B|}{A} 100$, where A and $\mathrm{B}$ are the values obtained using the pH-meter and the combined predictive values of the dyes, respectively.
} 

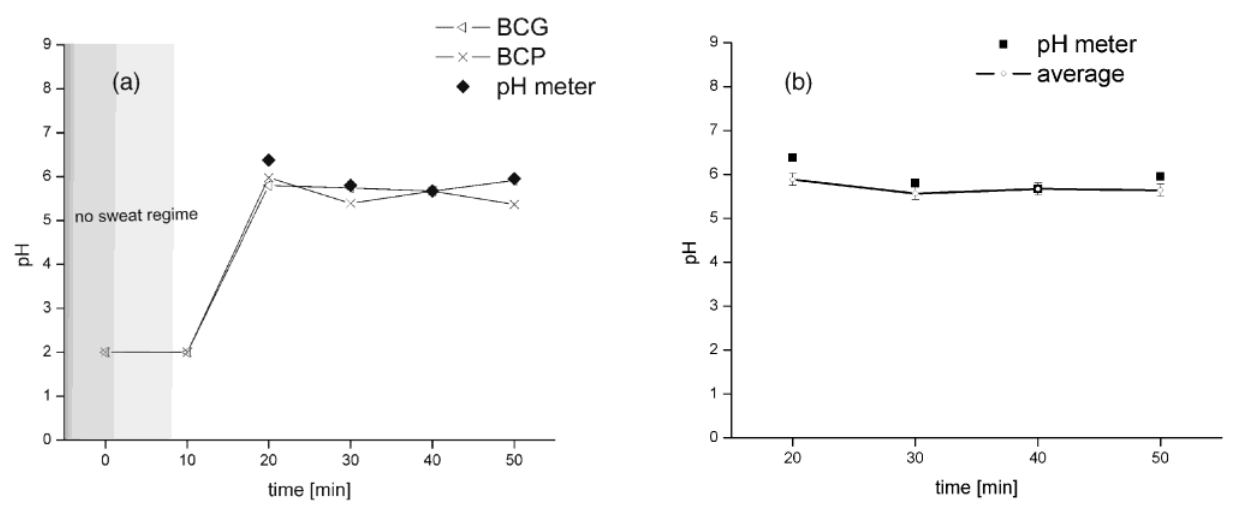

$<$ Fig. 7>

\section{Conclusions}

In this work, the fabrication, characterisation and the performance of a wearable, electronic-free and flexible micro-fluidic system based on ionic liquid polymer gels (ionogels) for monitoring in real-time the $\mathrm{pH}$ of the sweat generated during an exercise period has been presented.

As proven before, the ionogel matrix is very robust even at harsh $\mathrm{pH}$ conditions and that the $\mathrm{pH}$ indicators bromophenol blue, bromocresol green, bromocresol purple and bromothymol blue retained their $\mathrm{pH}$ indicator properties on the ionogel. The ionogeldye interactions ensure no leaching of the dyes during experiments, providing long durability of the device and accuracy on the $\mathrm{pH}$ of sweat measurements over time. The approach presented here provides immediate feedback regarding sweat composition, i.e. $\mathrm{pH}$, to individuals during exercise period. A particular colour pattern of the barcode corresponds to a defined $\mathrm{pH}$ of the sweat with an accuracy of $\sim 0.49 \mathrm{pH}$ units after applying standard image processing and analysis techniques to the pictures, which were captured during exercise trials when the sensor was applied on the skin. Future work will focus on the development of a more robust code for image processing, aiming a better resolution and accuracy in the $\mathrm{pH}$ prediction. Moreover, through a systematic comparison and correlation of $\mathrm{pH}$ of sweat with $\mathrm{pH}$ and lactate from blood, it will provide an easy, non-invasive and cheap tool to perform $\mathrm{pH}$ sweat analysis, improving sport performance and health.

\section{Acknowledgements}


This work was supported by Science Foundation Ireland under grant 07/CE/I1147 and the Research Career Start Programme 2010 fellowship form Dublin City University.

\section{Notes and references}

[1] Diamond D, Coyle S, Scarmagnani S, Hayes J (2008) Wireless sensor networks and chemo/biosensing. Chem Rev 108 (2):652-679

[2] Beauchamp M, Lands LC (2005) Sweat-testing: A review of current technical requirements. Pediatr Pulmonol 39 (6):507-511

[3] Jonsdottir B, Bergsteinsson H, Baldursson O (2008) Cystic Fibrosis - Review. Laeknabladid 94 (12):831-837

[4] Weber J, Kumar A, Kumar A, Bhansali S (2006) Novel lactate and pH biosensor for skin and sweat analysis based on single walled carbon nanotubes. Sens Actuators B 117 (1):308-313

[5] Casa DJ, Armstrong LE, Hillman SK, Montain SJ, Reiff RV, Rich BSE, Roberts WO, Stone JA (2000) National Athletic Trainers' Association position statement: Fluid replacement for athletes. J Athl Train 35 (2):212-224

[6] Schmid-Wendtner MH, Korting HC (2006) The pH of the skin surface and its impact on the barrier function. Skin Pharmacol Physiol 19 (6):296-302

[7] Patterson MJ, Galloway SDR, Nimmo MA (2002) Effect of induced metabolic alkalosis on sweat composition in men. Acta Physiol Scand 174 (1):41-46

[8] Granger D, Marsolais M, Burry J, Laprade R (2003) Na+/H+ exchangers in the human eccrine sweat duct. Am J Physiol-Cell Physiol 285 (5):C1047-C1058

[9] Patterson MJ, Galloway SDR, Nimmo MA (2000) Variations in regional sweat composition in normal human males. Exp Physiol 85 (6):869-875

[10] Morgan RM, Patterson MJ, Nimmo MA (2004) Acute effects of dehydration on sweat composition in men during prolonged exercise in the heat. Acta Physiol Scand 182 (1):37-43

[11] Whitesides G (2010) Solving problems. Lab Chip 10 (18):2317-2318

[12] Benito-Lopez F, Coyle S, Byrne R, Smeaton A, O'Connor NE, Diamond D (2009) Pump Less Wearable Microfluidic Device for Real Time $\mathrm{pH}$ Sweat Monitoring. Procedia Chem 1:1103-1106

[13] Diamond D (2004) Internet-scale sensing. Anal Chem 76 (15):278A-286A

[14] O'Toole M, Shepherd R, Wallace GG, Diamond D (2009) Inkjet printed LED based pH chemical sensor for gas sensing. Anal Chim Acta 652 (1-2):308-314

[15] Smyth C, Lau KT, Shepherd RL, Diamond D, Wu Y, Spinks GM, Wallace GG (2008) Selfmaintained colorimetric acid/base sensor using polypyrrole actuator. Sens Actuators B 129 (2):518-524 [16] Safavi A, Maleki N, Bagheri M (2007) Modification of chemical performance of dopants in xerogel films with entrapped ionic liquid. J Mater Chem 17 (17):1674-1681

[17] Seddon K (2002) Ionic liquids: designer solvents for green synthesis. TCE (730):33-35

[18] Byrne R, Coleman S, Fraser KJ, Raduta A, MacFarlane DR, Diamond D (2009) Photochromism of nitrobenzospiropyran in phosphonium based ionic liquids. Phys Chem Chem Phys 11 (33):72867291

[19] Byrne R, Fraser KJ, Izgorodina E, MacFarlane DR, Forsyth M, Diamond D (2008) Photo- and solvatochromic properties of nitrobenzospiropyran in ionic liquids containing the [NTf2]- anion. Phys Chem Chem Phys 10 (38):5919-5924

[20] Coleman S, Byrne R, Minkovska S, Diamond D (2009) Investigating Nanostructuring within Imidazolium Ionic Liquids: A Thermodynamic Study Using Photochromic Molecular Probes. The J Phys Chem B 113 (47):15589-15596

[21] Rogers RD, Seddon KR, Editors (2002) Ionic Liquids: Industrial Applications for Green Chemistry. (Proceedings of a Symposium held 1-5 April 2001 in San Diego, California.) [In: ACS Symp. Ser., 2002; 818.

[22] Wasserscheid P (2003) Recent developments in using ionic liquids as solvents and catalysts for organic synthesis. Organic Synthesis Highlights V:105-117

[23] Benito-Lopez F, Byrne R, Raduta AM, Vrana NE, McGuinness G, Diamond D (2010a) Ionogelbased light-actuated valves for controlling liquid flow in micro-fluidic manifolds. Lab Chip 10 (2):195201

[24] Neouze MA, Le Bideau J, Gaveau P, Bellayer S, Vioux A (2006) Ionogels, new materials arising from the confinement of ionic liquids within silica-derived networks. Chem Mater 18 (17):3931-3936 [25] Tamada M, Watanabe T, Horie K, Ohno H (2007) Control of ionic conductivity of ionic liquid/photoresponsive poly( amide acid) gels by photoirradiation. Chem Commun:4050-4052 
[26] Benito-Lopez F, Coyle S, Byrne R, O'Toole C, Barry C, Diamond D (2010b) Simple Barcode System Based on Inonogels for Real Time $\mathrm{pH}$-Sweat Monitoring. In: 7th International Workshop on Wearable and Implantable Body Sensor Networks (BSN 2010), 7-9 June 2010. pp 291-296

[27] Curto VF, Coyle S, Byrne R, Angelov N, Diamond D, Benito-Lopez F (2011) Concept and Development of an Autonomous Wearable Micro-fluidic Platform for real time $\mathrm{pH}$ Sweat Analysis, Sens Actuators B, Accepted.

[28] Ramnial T, Taylor S A, Bender M L, Gorodetsky B, Lee P T K, Dickie D A, McCollum B M, Pye C C, Walsby C J, Clyburne J A C (2008) Carbon-Centered Strong Bases in Phosphonium Ionic Liquids. J. Org. Chem. 73(3): 801-812

[29] O'Neill S, Conway S, Twellmeyer J, Egan O, Nolan K, Diamond D (1999) Ion-selective optode membranes using 9-(4-diethylamino-2-octadecanoatestyryl)-acridine acidochromic dye. Anal Chim Acta 398 (1):1-11

[30] MacFarlane DR, Pringle JM, Johansson KM, Forsyth SA, Forsyth M (2006) Lewis base ionic liquids. Chem. Commun. 1905-1917.

[31] Soller BR (1994) Design of intravascular fiber optic blood-gas sensors. IEEE Eng. Med. Biol. Mag. 13 (3): 327-335.

[32] A.I.S. Department of Sports Nutrition Data Sheet (2004) How much do athletes sweat? 1-5

[33] Morris D, Coyle S, Wu YZ, Lau KT, Wallace G, Diamond D (2009) Bio-sensing textile based patch with integrated optical detection system for sweat monitoring. Sens Actuators B 139 (1):231-236

[34] Byrne L, Lau K, Edwards S, Diamond D (2001) Digital imaging as a detector for quantitative colorimetric analyses, Proc. SPIE 4205, 267, doi:10.1117/12.417460. 


\section{Biographies}

Vincenzo Fabio Curto studied chemical engineering at University of Palermo, Italy (MSc Hons 2010). In 2010 he joined the Adaptive Sensors Group at Dublin City University where she is currently pursuing a PhD degree under the supervision of Prof. Dermot Diamond and Dr. Fernando BenitoLopez. His research interests include the development of wearable micro-fluidic system to perform real-time analysis.

Cormac Fay received a B.Eng. degree in Mechatronics and a M.Eng. degree in Telecommunications engineering, both from Dublin City University (DCU), Ireland, in 2005 and 2007, respectively. He subsequently pursued an internship within the Adaptive Information Cluster (AIC) in 2007 before progressing towards a research assistant position as part of the AIC and soon afterwards with the CLARITY Research Centre, DCU. His research area includes a range of disciplines including: novel environmental monitoring techniques, ultra low-power low cost environmental chemical sensing platforms, end-to-end system architectures, vision systems, wearable sensors, robotics, etc. His position continuously demands realizing the transition from chemical sensing to information retrieval via the world-wide-web.

Shirley Coyle is a researcher/designer in the field of wearable technologies and smart textiles. She has combined expertise in Biomedical Engineering and Fashion Design. She received her BEng in Electronic Engineering in 2000 from Dublin City University, Ireland. She then worked in the Information and Communications division in Siemens Ltd. for 2 years before commencing a Ph.D. study to develop the first optical brain computer interface. She received her PhD from the National University of Ireland Maynooth in 2005. Studying by night she graduated from the Grafton Academy of Fashion Design in 2008. She has worked on the EU FP6 'Biotex' project, a European-wide multipartner research effort to merge sensing capabilities with fabrics and textiles. She currently works within CLARITY: Centre for Sensor Web Technologies investigating ways to improve personal health and fitness using textile technologies.

Fernando Benito López studied chemistry at the Universidad Autonoma de Madrid and completed his master studies in the Department of Inorganic Chemistry in 2002. He obtained his PhD at the University of Twente, The Netherlands, under the supervision of Prof. David N. Reinhoudt and Dr. Willem Verboom in 2007. He carried out his postdoctoral research in the group of Prof. Dermot Diamond at Dublin City University, Dublin, Ireland. From 2010, he is Team Leader in polymer microfluidics at CLARITY: Centre for Sensor Web Technology, National Centre for Sensor Research, Dublin City University.

Robert Byrne studied pure and applied chemistry at Dublin City University (BSc Hons 2004) and received his PhD from Dublin City University (Materials Chemistry, 2008). His research interests include controlling molecular actuation by external stimuli for biomimetic applications.

Dermot Diamond received his PhD and DSc from Queen's University Belfast (Chemical Sensors, 1987, Internet Scale Sensing, 2002), and was VP for Research at Dublin City University (2002-2004). He has published over 200 peer-reviewed papers in international journals, is a named inventor in 13 patents, and is co-author and editor of three books. He is currently director of the National Centre for Sensor Research (www.ncsr.ie) and a Principle Investigator in CLARITY (www.clarity-centre.com/), a major research initiative focused on wireless sensor networks. In 2002 he was awarded the inaugural silver medal for Sensor Research by the RSC, London.

*Corresponding author: Tel.: +353 17007603 fax: +3531 7007995 .

E-mail addresses: fernando.lopez@dcu.ie (F. Benito-Lopez), vincenzo.curto2@mail.dcu.ie (V.F.Curto), dermot.diamond@dcu.ie (D. Diamond). 


\section{Figure Captions}

Fig. 1. (a) Picture of the micro-fluidic platform. (b) Micro-fluidic platform fabrication process.

Fig. 2. The molecular structure of the two components that make up the ionogel material. (a) $\mathrm{N}$ isopropylacrylamide and $N, N$-methylene-bis(acrylamide) crosslinked polymer in the ratio 100(x):5(y), and (b) the ionic liquid trihexyltetradecyl- phosphonium dicyanoamide $\left[\mathrm{P}_{6,6,6,14}\right][\mathrm{dca}]$ structure.

Fig. 3. Photographs of the micro-fluidic system at different pH's tested with artificial sweat (ISO 3160 2).

Fig. 4. Picture of the micro-fluidic system integrated into a plaster (a) and into a wrist-band (b).

Fig. 5. a) Schematic representation of the micro-fluidic system's performance over time. b) Series of pictures showing the channel performance in the micro-fluidic system (artificial sweat with dye), Pictures like these were used to estimate the sweat flow rate through the device.

Fig. 6. Calibration curves showing $\mathrm{pH} v \boldsymbol{s} \cdot \mathrm{R}^{\prime}=\mathrm{R} /(\mathrm{R}+\mathrm{G}+\mathrm{B})$ normalised $[0,1]$ a) bromocresol green and b) bromocresol purple.

Fig. 7. $\mathrm{pH}$ determination of sweat using the micro-fluidic system during a 50 min training period. (a) Plot showing the reference instrument in conjunction with the individual predictions of each dye when normalised with respect to the reference patches and predicted using the calibration model. (b) Plot of the reference measurement and the average of all the dye predictions when weighted equally. 